\title{
Semiclassical Study of Quantum Scattering on the Line
}

\author{
Thierry Ramond \\ Laboratoire Analyse Géométrie et Applications (URA CNRS 742), Université Paris Nord, \\ F-93430 Villetaneuse, France. e-mail: ramond@math.univ-paris13.fr
}

Received: 28 December 1994/ in revised form: 20 June 1995

\begin{abstract}
We study the well-known problem of 1-d quantum scattering by a potential barrier in the semiclassical limit. Using the so-called exact WKB method and semiclassical microlocal analysis techniques, we get a very precise and complete description of the scattering matrix, in particular when the energy is very close to a unique, quadratic maximum of the potential. In our one-dimensional setting, we also recover the Bohr-Sommerfeld quantization condition for the resonances generated by such a maximum.
\end{abstract}

\section{Introduction}

This paper is devoted to the semiclassical study of quantum scattering by a potential barrier in dimension 1 , in particular in the transition regime where the energy is very close to the top of the barrier. Many articles have been written since the 30's dealing with the computation of the transmission coefficient through a barrier, and this problem is one of the starting points for the development of what is nowadays called JWKB method. The exponential decay of the transmission coefficient (cf. Theorem 1 below) has been known since the first papers by R.E.Langer and H.Jeffreys (see e.g. [La] and [Je]), by the use of the famous connection formulae. Other techniques have been developed during the 60's, in particular by N.Fröman and P.O.Fröman (see [Fr-Fr]), M.V.Fedoryuk (see [Fe]) and F.W.J.Olver (see [Ol1]). Their works were based on a JWKB-like approximation method for the solutions of a 1-dimensional Schrödinger equation in the complex plane, often known as phase integral method, which has been recently improved by J.Ecalle and A.Voros (cf. [Ec, Vo]) and used in a new formalism by A.Grigis for the study of Hill's equation (cf. [Gr]). The new fact in what is now usually called exact-WKB analysis is that it provides, rather than approximate solutions with error bounds, exact solutions with a complete asymptotic expansion with respect to the semiclassical parameter $h$ for example, with a priori estimates on the coefficients. A huge amount of papers has been written on this subject, and it is a difficult job to identify even the main contributions. We think that we have not forgoten too many important names, but the reader should refer to the 
books [Hd, Fr-Fr, Ol2], where he would find many references as well as detailed and interesting historical discussions.

We have written in the first part of this article the exact-WKB analysis for the 1-dimensional scattering Schrödinger operator, and obtained results which, at least in the barrier-penetration case, are very familiar (see for example Eq. (10.17) in [O12], Eq. (9.12) in [Fr-Fr] or Eq. (1.15) in [Fe]). The method also looks very much like the one developed in Chap. 13, Part 15 of [O12] or the general theory developed in [Ev$\mathrm{Fe}$, but there is something new in our results even in that case: we show a complete asymptotic expansion with a priori estimates on the coefficients. In the second part we have studied the semiclassical behavior of the scattering matrix for energies which are very near (in the semiclassical sense) to the critical value of a unique quadratic maximum of the potential. It has been already noticed that the exact-WKB method does not give good enough estimates to treat the case where two simple turning points coalesce as $h$ goes to 0 (see [Ge-Gr]). We used here, together with the exact-WKB constructions, microlocal techniques which have been introduced by B.Helffer and J.Sjöstrand for the study of Harper's equation (see in particular [He-Sj]). In particular we performed as in [Mä] and [Ra] a microlocal reduction to a branching operator which is closely related to Weber's equation. Such techniques have also been used in a $\mathscr{C}^{\infty}$ setting in $[\mathrm{Sj} 3]$, and also in [Co-Pa] where a more geometrical discussion can be found. As also noticed in [As-Du] or [Na], the point is that a tunnel effect in the phase-space occurs, which can be described analytically for the branching model. We have obtained that way a very precise description of the scattering matrix at the critical energy level which is new as far as we know. Thanks to its sharpness, we also recover a result about quantization of the resonances lying near a quadratic maximum which have been proven independently in the n-dimensional case by J.Sjöstrand (see [Sj1]) and P.Briet, J.-M.Combes and P.Duclos (see [Br-Co-Du]). We shall come back in forthcoming papers with S.Fujiie to a more global study of resonances in these settings.

Let us describe now the scattering problem briefly. We denote by $P(x, h D)=$ $h^{2} D^{2}+V(x)$ the 1-dimensional Schrödinger operator (with $D=-i \partial_{x}$ ), where the potential $V(x)$ is smooth and goes to 0 sufficiently fast as $x$ goes to infinity. Because of this fall-off at infinity, the solutions of the Schrödinger equation

$$
P u=E u,
$$

where $E$ is a real parameter, should behave as $x \rightarrow \pm \infty$ like

$$
a_{+}^{l, r}(E, h) \mathrm{e}^{i \sqrt{E} x / h}+a_{-}^{l, r}(E, h) \mathrm{e}^{-i \sqrt{E} x / h},
$$

where $l$ and $r$ stand for left and right and correspond respectively to $x \rightarrow-\infty$ and $x \rightarrow+\infty$. The four Jost solutions $f_{ \pm}^{l, r}$ are the solutions which behave exactly as $\mathrm{e}^{i \sqrt{E} x / h}$ or $\mathrm{e}^{-i \sqrt{E} x / h}$ as $x \rightarrow \pm \infty$. The reflection or scattering problem is the following: what are the components of a solution $u$ of the Schrödinger equation in the basis $\left(f_{+}^{r}, f_{-}^{l}\right)$ of the outgoing Jost solutions, knowing its components in the basis $\left(f_{+}^{l}, f_{-}^{r}\right)$ of the incoming Jost solutions. The 2 by 2 matrix relating these coefficients is called the scattering matrix and we will denote it by

$$
\mathbb{S}(E, h)=\left(\begin{array}{ll}
s_{11} & s_{12} \\
s_{21} & s_{22}
\end{array}\right) .
$$


The reflection and transmission coefficients $R(E, h)$ and $T(E, h)$ are by definition the square of the modulus of the coefficients $s_{21}$ and $s_{11}$ respectively. They correspond to the probability for a purely incoming-from-the-left particle $\left(1 . f_{+}^{l}+0 . f_{-}^{r} \ldots\right)$ to be reflected to the left or transmitted to the right $\left(=s_{21} f_{-}^{l}+s_{11} f_{+}^{r}\right)$. We shall study the behavior of these quantities in different regions of the $(h, E)$-plane as $h \rightarrow 0$, under the following general hypothesis on the potential $V$ :

(A) The function $V$ is real on the real axis, analytic in the sector

$$
\mathscr{S}=\{x \in \mathbb{C},|\operatorname{Im} x|<\delta|\operatorname{Re} x|+\eta\}
$$

for some $\delta, \eta>0$, and satisfies the following estimates:

$$
V(x)=O\left(\frac{1}{|x|^{r}}\right)
$$

for some $r>1$ as $|x|$ goes to infinity in $\mathscr{S}$.

We will show that under this assumption the Jost solutions exist (see discussion at the end of Sect. 3 for the long-range case, that is when $r \in] 0,1]$ ). Because $V$ is real on the real axis, we have $\left(f_{+}^{l, r}\right)^{*}=f_{-}^{l, r}$, where $u^{*}(x)=\overline{u(\bar{x})}$, and the scattering matrix $\mathbb{S}(E, h)$ is unitary. We also have the following relations between the coefficients of $\mathbb{S}(E, h)$ :

$$
s_{11}(E, h)=s_{22}(E, h) \quad \text { and } \quad s_{12}(E, h)=-\bar{s}_{21}(E, h) \frac{s_{11}(E, h)}{\bar{s}_{11}(E, h)},
$$

so that $s_{11}$ and $s_{21}$ determine completely the scattering matrix. We also have the well-known relation $R(E, h)+T(E, h)=1$.

We have first studied the case where the energy level $E$ is far below the extrema of $V$ on the real axis, that is the distance between $E$ and any extrema does not vanish as $h$ does. We have obtained the following

Theorem 1. Transmission through a barrier. Suppose the potential $V$ satisfies assumptions (A). Let $V_{m}$ be the lowest local extremum of $V$ on the real axis. Suppose $C<E<V_{m}-C$ for some constant $C>0$ independent of $h$, and let $a(E)<b(E)$ be the only two real zeros of $V(x)-E$ (see Fig. 1). If these zeros are simple, there exists two classical analytic symbols $\phi_{1}(h)$ and $\phi_{2}(h)$ of non-negative order (see Definition 1 below) such that

$$
\left\{\begin{array}{l}
s_{11}(E, h)=\left(1+h \phi_{1}(h)\right) \exp \left\{-\frac{S(E)}{h}\right\} \exp \left\{i \frac{T(E)}{h}\right\} \\
s_{21}(E, h)=i\left(1+h \phi_{2}(h)\right) \exp \left\{\frac{2 i}{h}\left(\sqrt{E} a+\int_{-\infty}^{a} Q(t, E) d t\right)\right\},
\end{array}\right.
$$

where $S(E)$ is the classical action between the two turning points $a(E)$ and $b(E)$

$$
S(E)=\int_{a(E)}^{b(E)} \sqrt{V(x)-E} d x
$$

We have also written $Q(t, E)=\sqrt{E-V(t)}-\sqrt{E}$ for $t<a(E)$ and

$$
T(E)=-\sqrt{E}(b(E)-a(E))+\int_{b(E)}^{+\infty} Q(t, E) d t+\int_{-\infty}^{a(E)} Q(t, E) d t .
$$



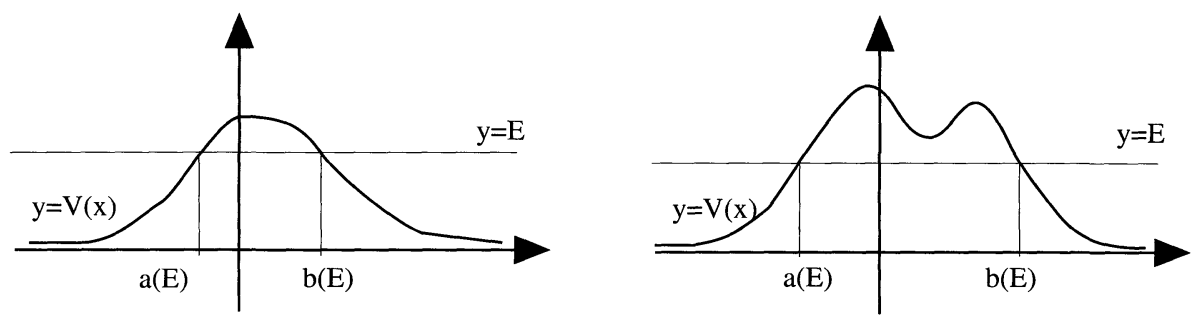

Fig. 1. Transmission through a barrier

We have studied more closely what happens in the neighborhood of a maximum of the potential under the following assumption on $V$ :

(B) The function $V$ has a unique maximum on the real axis at $x=0$ with $V(0)=V_{0}$ and $V^{\prime \prime}(0)=-k^{2}$ for a real strictly positive $k$.

When $V$ satisfies assumptions (A) and (B), and for energies $E$ close enough to $V_{0}$, there exist in a complex neighborhood of 0 exactly two simple turning points which are real and of opposite sign for $E<V_{0}$, close to $\pm \frac{1}{k} \sqrt{2\left(V_{0}-E\right)}$. They are complex-conjugate for $E>V_{0}$, close to $\pm \frac{\imath}{k} \sqrt{2\left|V_{0}-E\right|}$. We define then the classical action $S(E)$ by

$$
S(E)=\frac{1}{2} \oint_{\gamma}(V(x)-E)^{1 / 2} d x,
$$

where $\gamma$ is the boundary (oriented counterclockwise) of a disk in $\mathscr{S}$ which contains the turning points. The determination of the square root is fixed so that $(V(x)-E)^{1 / 2}$ belongs to $i \mathbb{R}^{+}$for the real positive $x$ on $\gamma$. Notice that we have

$$
S(E)= \begin{cases}\int_{a(E)}^{b(E)} \sqrt{V(t)-E} d t & \text { when } E<V_{0} \\ i \int_{a(E)}^{b(E)} \sqrt{|V(t)-E|} d t & \text { when } E>V_{0}\end{cases}
$$

and that $S(E)$ is real, positive when $E<V_{0}$ and negative for $E>V_{0}$. We shall also use the following notations:

$$
T(E)= \begin{cases}\sqrt{E}(a(E)-b(E))+\int_{-\infty}^{a(E)} Q(t, E) d t+\int_{b(E)}^{+\infty} Q(t, E) d t & \text { for } E<V_{0} \\ \int_{-\infty}^{+\infty} Q(t, E) d t+i \int_{a(E)}^{b(E)}(V(t)-E)^{1 / 2} d t & \text { for } E>V_{0}\end{cases}
$$

and

$$
T_{-}(E)= \begin{cases}\sqrt{E} a(E)+\int_{-\infty}^{a(E)} Q(t, E) d t & \text { for } E<V_{0} \\ \int_{-\infty}^{0} Q(t, E) d t-i \int_{0}^{a(E)}(V(t)-E)^{1 / 2} d t & \text { for } E>V_{0}\end{cases}
$$

with $Q(t, E)=\sqrt{E-V(t)}-\sqrt{E}$ for real, large enough $t$. We can state now our main result: 
Theorem 2. Scattering at the top of a barrier. There exist a constant $C>0$ independent of $h$, three classical analytic symbols $m_{1}(E, h), m_{2}(E, h), m_{3}(E, h)$ of non-negative order, and a strictly positive real number $\epsilon$ such that for any $E \in$ ]$V_{0}-C, V_{0}+C[$, we have

$$
\left\{\begin{aligned}
s_{11}(E, h)= & \frac{1}{\sqrt{2 \pi}} \exp \left\{-\frac{S(E)}{2 h}+i \frac{T(E)}{h}\right\} \Gamma\left(\frac{1}{2}+i \frac{S(E)}{\pi h}\left(1+h^{2} m_{1}(h)\right)\right) \\
& \quad \exp \left\{-\frac{i S(E)}{\pi h}\left(\ln \frac{|S(E)|}{\pi h}-1\right)\right\}\left(1+h m_{2}(h)\right)+O\left(\mathrm{e}^{-\epsilon / h}\right) \\
s_{21}(E, h)= & \frac{i}{\sqrt{2 \pi}} \exp \left\{\frac{S(E)}{2 h}+2 i \frac{T-(E)}{h}\right\} \Gamma\left(\frac{1}{2}+i \frac{S(E)}{\pi h}\left(1+h^{2} m_{1}(h)\right)\right) \\
& \exp \left\{-\frac{i S(E)}{\pi h}\left(\ln \frac{|S(E)|}{\pi h}-1\right)\right\}\left(1+h m_{3}(h)\right)+O\left(\mathrm{e}^{-\epsilon / h}\right) .
\end{aligned}\right.
$$

It is interesting to compare this theorem with the first one. We show below that for $V_{0}-E$ small enough

$$
S(E) \sim \frac{\pi}{\sqrt{2} k}\left(V_{0}-E\right),
$$

so that $|S(E) / h| \rightarrow \infty$ provided $\left|V_{0}-E\right| / h \rightarrow \infty$. Thus we shall use Stirling's formula in the complex plane (see [O12] Chap. 8, Part 4), which can be written as

$$
\Gamma(z)=\sqrt{2 \pi} \exp \left\{-z+(z-1 / 2) \ln (z)+\frac{f(z)}{z}\right\},
$$

where $f(z)$ is analytic and bounded function in the domain $\{z \in \mathbb{C},|z|>R,|\arg z|<$ $\pi-\delta\}$ for any large enough $R$ and any $\delta>0$. If we notice that for any real $M$,

$$
\ln \left(\frac{1}{2}+i M\right)=\ln (|M|)+i \frac{\pi}{2} \operatorname{sgn}(M)-\frac{i}{2 M}+\left(\frac{1}{M}\right)^{2} g(1 / M),
$$

where $g$ is analytic near the origin, Eq. (2) gives, for some analytic and bounded function $k$,

$$
\Gamma\left(\frac{1}{2}+i M\right)=\sqrt{2 \pi} \exp \left\{-\frac{\pi|M|}{2}+i M(\ln |M|-1)+\frac{i}{M} k(1 / M)\right\} .
$$

Under assumption (B) and for energies $E$ such that $C<E<V_{0}$ and $\left(V_{0}-E\right) / h \rightarrow$ $+\infty$ as $h \rightarrow 0$, Theorem 2 and Eq. (3) with $M=S(E) / \pi h$ give exactly the formulas of Theorem 1.

Equation (3) and Theorem 2 give also an interesting result in the case where $C>E>V_{0}$ and $\left(V_{0}-E\right) / h \rightarrow+\infty$ as $h \rightarrow 0$. Then $S(E)$ is negative and we have

Theorem 3. Reflection over a potential barrier. We suppose the potential $V$ satisfies assumptions $(A)$ and $(B)$. We also suppose that $V_{0}+C_{1}<E<C$ for some constant $C_{1}>0$ independent of $h$. Then there exists two classical analytic symbols $\phi_{1}(h)$ and $\phi_{2}(h)$ of non-negative order (see Definition 1 below) such that

$$
\left\{\begin{array}{l}
s_{11}(E, h)=\left(1+h \phi_{1}(h)\right) \exp \left\{\frac{i}{h} \int_{-\infty}^{+\infty} Q(t, E) d t\right\} \\
s_{21}(E, h)=i\left(1+h \phi_{2}(h)\right) \exp \left\{\frac{2 i}{h} \int_{-\infty}^{0} Q(t, E) d t+\frac{2}{h} \int_{0}^{b(E)}(V(t)-E)^{1 / 2} d t\right\}
\end{array}\right.
$$

where we have written again $Q(t, E)=\sqrt{E-V(t)}-\sqrt{E}$. 


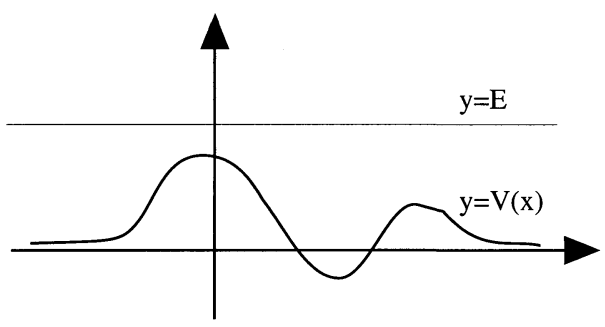

Fig. 2. Reflection over a barrier

Notice that we have not written here the exponentially small error term which should come from Theorem 2. In fact we have proven this more precise result in Sect. 4 by the mean of the exact WKB approach, and we have shown there that this theorem is still true under a more general hypothesis: the important point is that the geometrical configuration of the Stokes lines for the $E$ we consider should be the same as in the case where assumption (B) holds (see Proposition 7).

Concerning the resonances generated by a quadratic maximum, our microlocal constructions give also

Theorem 4. Bohr-Sommerfeld quantization condition for the resonances generated by a quadratic maximum. Suppose $V$ satisfies assumptions $(A)$ and $(B)$. There exists a constant $C>0$ depending only on the potential such that we have the following quantization condition for the resonant energies lying in the half-disk $\left\{\left|V_{0}-E\right|<\right.$ $C h\} \cap\{\operatorname{Im} E<0\}$ :

$$
S(E)=i(2 k+1) \frac{\pi h}{2}+O\left(h^{2}\right), k \in \mathbb{N}
$$

where $S(E)$ is the analytic continuation of the classical action defined by Eq. (1) to this half disk.

This paper is organized as follows: Part $\mathbf{1}$ is this introduction. We recall in the Second part how the exact-WKB method permits to construct exact solutions of the Schrödinger equation and give their asymptotic expansions in bounded domains of $\mathscr{S}$. In the Third part we adapt this method in order to obtain the Jost solutions and their asymptotic expansions as $h \rightarrow 0$. Part 4 is devoted to the proof of the first theorem using the constructions of Part 3 . We also mention other results concerning the over-barrier case. In Part 5 we construct microlocal solutions near a quadratic maximum of the potential, recalling first the reduction theorem and studying precisely the branching model. The Sixth part ends the proof of the last results, connecting the Jost solutions of Part 2 and the microlocal solutions of Part 5. At last we have put together in a brief Appendix the minimal set of notions in semiclassical microlocal analysis that we hope makes this text understandable (one may find more details in [Sj2], [He-Sj], or [De]). 


\section{Exact WKB Constructions}

We construct here solutions of the Schrödinger equation

$$
-h^{2} u^{\prime \prime}+V(x) u=E u
$$

in bounded domains of the sector $\mathscr{S}$ using the exact WKB method. First of all we make the change of variables $x \mapsto z$ given by

$$
x \mapsto z\left(x, x_{0}, E\right)=\int_{\gamma\left(x_{0}, x\right)}(V(t)-E)^{1 / 2} d t .
$$

One of our tasks will be of course to study the subsets of $\mathscr{S}$ where this function is well-defined, but let's work formally for a while. Writing a solution $u(x, E, h)$ as

$$
u(x, E, h)=(V(x)-E)^{-1 / 4} g\left(z\left(x, x_{0}, E\right), E, h\right),
$$

we obtain the following equation for $g(z)$ :

$$
-g^{\prime \prime}+\left(H^{2}-H^{\prime}+\frac{1}{h^{2}}\right) g=0
$$

where $H(z)$ is given by

$$
H(z)=-\frac{1}{4} \frac{V^{\prime}(x(z, E))}{(V(x(z, E))-E)^{3 / 2}}=(V(x)-E)^{-1 / 4} \partial_{x}(V(x)-E)^{-1 / 4}
$$

as soon as $x(z, E)$ is well-defined in $\Omega(E)$. Now we put

$$
g_{ \pm}(z, E, h)=\mathrm{e}^{ \pm z / h} W_{ \pm}(z, E, h)
$$

and obtain

$$
\left(H^{2}-H^{\prime}\right) W_{ \pm}= \pm \frac{2}{h} W_{ \pm}^{\prime}+W_{ \pm}^{\prime \prime}
$$

One then sees easily that the series $W_{ \pm}=\sum_{n \geq 0} W_{n, \pm}$, given by

$$
\left\{\begin{aligned}
W_{0, \pm} & =1 \\
\left(\partial_{z} \pm \frac{2}{h}\right) W_{2 p+1, \pm} & =-H W_{2 p, \pm} \\
\partial_{z} W_{2 p, \pm} & =-H W_{2 p-1, \pm}
\end{aligned}\right.
$$

is a formal solution of Eq. (6). So we investigate the solutions of this system. Suppose $\tilde{\Omega}$ is an open simply-connected domain of the $z$-plane, where $z \mapsto H(z)$ is welldefined and analytic. Then Eq. (7) defines a sequence $\left(W_{n, \pm}\right)$ of analytic functions in $\tilde{\Omega}$ which is unique up to some arbitrary constants. We fix these constants choosing a base point $\tilde{z}$ in $\tilde{\Omega}$ and consider the sequence $\left(W_{n, \pm}(z, h, \tilde{z})\right)$ satisfying Eq. (7) and

$$
W_{n, \pm}(\tilde{z}, h, \tilde{z})=0, n \geq 1 \text {. }
$$

One immediately gets the corresponding integral equations 


$$
\left\{\begin{aligned}
W_{0, \pm}(z, h, \tilde{z}) & =1 \\
W_{2 p+1, \pm}(z, h, \tilde{z}) & =-\int_{\Gamma(\tilde{z}, z)} \mathrm{e}^{ \pm 2(\zeta-z) / h} H(\zeta) W_{2 p, \pm}(\zeta) d \zeta \\
W_{2 p, \pm}(z, h, \tilde{z}) & =-\int_{\Gamma(\tilde{z}, z)} H(\zeta) W_{2 p-1, \pm}(\zeta) d \zeta
\end{aligned}\right.
$$

where $\Gamma(\tilde{z}, z)$ is any path in $\tilde{\Omega}$ beginning at $\tilde{z}$ and ending at $z$. The solutions of this system can be written as (for $p \geq 1$ )

$$
\begin{aligned}
& W_{2 p-1}(z, h, \tilde{z})= \\
& \quad-\int_{\Gamma_{2 p-1}^{ \pm}(\tilde{z}, z)} \mathrm{e}^{ \pm 2\left(\zeta_{1}-\zeta_{2}+\cdots+\zeta_{2 p-1}-z\right) / h} H\left(\zeta_{1}\right) \ldots H\left(\zeta_{2 p-1}\right) d \zeta_{1} \ldots d \zeta_{2 p-1}
\end{aligned}
$$

and

$$
\begin{aligned}
& W_{2 p}(z, h, \tilde{z})= \\
& \quad \int_{\Gamma_{2 p}^{ \pm}(\tilde{z}, z)} \mathrm{e}^{ \pm 2\left(\zeta_{1}-\zeta_{2}+\cdots+\zeta_{2 p-1}-\zeta_{2 p}\right) / h} H\left(\zeta_{1}\right) \ldots H\left(\zeta_{2 p}\right) d \zeta_{1} \ldots d \zeta_{2 p}
\end{aligned}
$$

where $\Gamma_{n}^{ \pm}(\tilde{z}, z)$ is the set of $n$-tuples of points $\left(\zeta_{1}, \ldots, \zeta_{n}\right)$ put in increasing order on the path $\Gamma^{ \pm}(\tilde{z}, z)$. The corresponding series

$$
W_{ \pm}(z, h, \tilde{z})=\sum_{n \geq 0} W_{n, \pm}(z, h, \tilde{z})
$$

will converge in $\tilde{\Omega}$ provided for example that for all $z \in \Omega$ there exits a path $\Gamma^{ \pm}(\tilde{z}, z)$ of finite length $L$ and a constant $A^{ \pm}(h)>0$ such that

$$
\sup _{\zeta \in \Gamma^{ \pm}}\left|\mathrm{e}^{2 \zeta / h} H(\zeta)\right| \leq A^{ \pm}(h) \quad \text { and } \sup _{\zeta \in \Gamma^{ \pm}}\left|\mathrm{e}^{-2 \zeta / h} H(\zeta)\right| \leq A^{ \pm}(h)
$$

because the Volterra equations above will then give, for $n \geq 1$,

$$
\left|W_{n, \pm}(z)\right| \leq \frac{\left(A^{ \pm}(h) L\right)^{n}}{n !}
$$

In particular one sees that the convergence is uniform in $\tilde{\Omega}$ when this set is bounded. In that case we can obtain asymptotic expansions in $h$ for the functions $W_{n, \pm}$ inside $\tilde{\Omega}$. We recall first the following

Definition 1. A function $f(z, h)$ defined in $U \times] 0, h_{0}[$, where $U$ is an open set in $\mathbb{C}$ and $h_{0}$ a real strictly positive number, is called a classical analytic symbol (CAS) of order $m \in \mathbb{N}$ in $h$ if $f$ is an analytic function of $z$ in $U$ and if there exists a sequence $\left(a_{j}(z)\right)$ of analytic functions in $U$ such that

- For all compact set $K \subset U$, there exists $C>0$ such that, for all $z$ in $K$, one has $\left|a_{j}(z)\right| \leq C^{j+1} j^{j}$.

- For all $z \in U, f(z, h)$ admits the series $\sum_{j \geq 0} a_{j}(z) h^{m+j}$ as asymptotic expansion as $h$ goes to zero. 
We reproduce now without proof a result that can be found in [Ge-Gr] (see Prop. 1.2 there). The reader should also look at Proposition 2 below where the same kind of result is proven in the case of a particular choice of base points.

Proposition 1. Let $\tilde{\Omega}$ be an open simply-connected bounded domain in $\mathbb{C}$ where the function $H(z)$ is analytic, $\tilde{z}$ a fixed point of $\tilde{\Omega}$ and $\tilde{\Omega}^{+}(\tilde{z})=\tilde{\Omega} \cap\{\operatorname{Re} z>\operatorname{Re} \tilde{z}\}$, $\tilde{\Omega}^{-}(\tilde{z})=\tilde{\Omega} \cap\{\operatorname{Re} z<\operatorname{Re} \tilde{z}\}$. The functions $W_{n,+}(z, h, \tilde{z})$ and $W_{n,-}(z, h, \tilde{z})$ are classical analytic symbols of order $\left[\frac{n+1}{2}\right]$ in $\tilde{\Omega}^{+}(\tilde{z})$ and $\tilde{\Omega}^{-}(\tilde{z})$ respectively. Moreover the functions $W_{ \pm}^{\text {even }}(z, h, \tilde{z})$ and $W_{ \pm}^{\text {odd }}(z, h, \tilde{z})$ given by

$$
W_{ \pm}^{\text {even }}(z, h, \tilde{z})=\sum_{n \geq 0} W_{2 n}^{ \pm}(z, h, \tilde{z}) \quad \text { and } \quad W_{ \pm}^{\text {odd }}(z, h, \tilde{z})=\sum_{n \geq 0} W_{2 n+1}^{ \pm}(z, h, \tilde{z})
$$

are classical analytic symbols of order 0 and 1 in $\tilde{\Omega}^{+}(\tilde{z})$ and $\tilde{\Omega}^{-}(\tilde{z})$ respectively.

We go back to the $x$-plane and use the discussion above to construct solutions of our equation in bounded domains of the sector $\mathscr{S}$. Let $\Omega(E)$ be an open, simplyconnected set of $\mathscr{S}$ with no turning point. We fix a determination of $t \mapsto(V(t)-E)^{1 / 4}$ in that set and define the function $z\left(x, x_{0}, E\right)$ as

$$
x \mapsto z\left(x, x_{0}, E\right)=\int_{x_{0}}^{x}(V(t)-E)^{1 / 2} d t,
$$

where $x_{0}$ is some point of $\Omega(E)$ and the integration is performed along any curve in $\Omega(E)$ going from $x_{0}$ to $x$. We introduce now the notions of canonical path and canonical set for the function $x \mapsto z$ :

Definition 2. Let $\tilde{x}$ and $x$ be points in $\Omega(E)$. We say that a path $\gamma(\tilde{x}, x)$ in $\Omega(E)$, starting at $\tilde{x}$ and finishing at $x$ is a canonical path of type \pm if the function $t \mapsto$ $\pm \operatorname{Re} z\left(t, x_{0}, E\right)$ is strictly increasing along $\gamma(\tilde{x}, x)$. We also define the canonical set $\Omega^{ \pm}(\tilde{x})$ of type \pm as the subset of $\Omega(E)$ for all points of which there exists a canonical path of type \pm going from $\tilde{x}$ to it.

It seems useful to mention here that the notion of canonical path, that we have borrowed in $[\mathrm{Ev}-\mathrm{Fe}]$ and $[\mathrm{Fe}]$, corresponds to the one of progressive path used by most of the authors. Our canonical set is the complementary of what is sometimes called a shadow zone (see [O12], Chap. 6, Part 11.4).

Let $\tilde{x}$ be a point in $\Omega(E)$. For all $x$ in the corresponding canonical set $\Omega^{ \pm}(\tilde{x})$ one can find a canonical path $\gamma(\tilde{x}, x)$ and consider its image $\Gamma(\tilde{z}, z)$ by $x \mapsto z$. Denoting by $\tilde{\Omega}^{ \pm}(\tilde{z})$ the image of $\Omega^{ \pm}(\tilde{x})$ by $x \mapsto z$, we see that we can perfectly define the sequence of function $\left(W_{n, \pm}\right)$ in that set using Eq. (8) and Eq. (9), where the integrals are performed along $\Gamma(\tilde{z}, z)$. Using these notations, we state the main result of this section:

Proposition 2. The function $w_{ \pm}$defined in $\Omega^{ \pm}(\tilde{x})$ by

$$
\begin{aligned}
& w_{ \pm}\left(x, E, h ; x_{0}, \tilde{x}\right)= \\
& \quad(V(x)-E)^{-1 / 4} \mathrm{e}^{ \pm z\left(x, x_{0}, E\right) / h} \sum_{n \geq 0} W_{n, \pm}\left(z\left(x, x_{0}, E\right), h, z\left(\tilde{x}, x_{0}, E\right)\right)
\end{aligned}
$$

is the solution of the following Cauchy problem: 


$$
\left\{\begin{array}{l}
-h^{2} u^{\prime \prime}+V(x) u=E u \\
w_{ \pm}\left(\tilde{x}, E, h ; x_{0}, \tilde{x}\right)=(V(\tilde{x})-E)^{-1 / 4} \mathrm{e}^{ \pm z\left(\tilde{x}, x_{0}, E\right) / h} \\
\partial_{x} w_{ \pm}\left(\tilde{x}, E, h ; x_{0}, \tilde{x}\right)= \pm \frac{1}{h}(V(\tilde{x})-E)^{1 / 4} \mathrm{e}^{ \pm z\left(\tilde{x}, x_{0}, E\right) / h}
\end{array}\right.
$$

Moreover the functions

$$
W_{ \pm}^{\text {even }}=\sum_{n \geq 0} W_{2 n, \pm}\left(z\left(x, x_{0}, E\right), h, z\left(\tilde{x}, x_{0}, E\right)\right)
$$

and

$$
W_{ \pm}^{\text {odd }}=\sum_{n \geq 0} W_{2 n+1, \pm}\left(z\left(x, x_{0}, E\right), h, z\left(\tilde{x}, x_{0}, E\right)\right)
$$

are classical analytic symbols in $\Omega^{ \pm}(\tilde{x})$ of order 0 and 1 respectively.

Proof. This result is almost a direct consequence of Proposition 1, noticing that the function $H(z)$ is well-defined and analytic in $\tilde{\Omega}^{ \pm}(\tilde{z})$. The only remaining thing to do is to compute the value of $\partial_{x} w_{ \pm}$at $\tilde{x}$. Using logarithmic derivatives, one can easily see that

$$
\begin{aligned}
& w_{ \pm}^{\prime}\left(x, E, h, x_{0}, \tilde{x}\right)= \\
& \quad \pm \frac{1}{h}(V(x)-E)^{1 / 4} \mathrm{e}^{ \pm z_{0}(x) / h}\left\{W_{ \pm}^{\text {even }}\left(z_{0}(x), h, \tilde{z}\right)-W_{ \pm}^{\text {odd }}\left(z_{0}(x), h, \tilde{z}\right)\right\}
\end{aligned}
$$

where $z_{0}(x)$ stands for $z\left(x, x_{0}, E\right)$ and $\tilde{z}$ for $z\left(\tilde{x}, x_{0}, E\right)$, and this ends the proof.

One of the main features of this construction is that the wronskians of two solutions can be easily calculated. We just recall that the wronskian $\mathscr{W}(u, v)(x)=u^{\prime}(x) v(x)-$ $u(x) v^{\prime}(x)$ of two solutions $u$ and $v$ of Eq. (4) doesn't depend on $x$ and is zero if and only if $u$ and $v$ are proportional. An obvious computation using Eq. (10) gives, with the same notations

Proposition 3. Let $x_{0}$ and $x_{1}$ be two points in $\Omega(E)$. If, for given $x_{+}$and $x_{-}$the canonical sets $\Omega^{+}\left(x_{+}\right)$and $\Omega^{-}\left(x_{-}\right)$have a non-empty intersection, then for any $x \in$ $\Omega^{+}\left(x_{+}\right) \cap \Omega^{-}\left(x_{-}\right)$one has

$$
\begin{gathered}
\mathscr{W}\left(w_{+}\left(., h, E ; x_{0}, x_{+}\right), w_{-}\left(., h, E ; x_{1}, x_{-}\right)\right)(x)= \\
\frac{2}{h} \mathrm{e}^{z_{0}\left(x_{1}\right) / h}\left\{W_{+}^{e v e n}\left(z_{0}(x), h, z_{+}\right) W_{-}^{\text {even }}\left(z_{1}(x), h, z_{-}\right)-\right. \\
\left.W_{+}^{\text {odd }}\left(z_{0}(x), h, z_{+}\right) W_{-}^{\text {odd }}\left(z_{1}(x), h, z_{-}\right)\right\} .
\end{gathered}
$$

If, for given $x_{+}$and $\tilde{x}_{+}$the canonical sets $\Omega^{+}\left(x_{+}\right)$and $\Omega^{-}\left(\tilde{x}_{+}\right)$have a non-empty intersection, then for any $x \in \Omega^{+}\left(x_{+}\right) \cap \Omega^{+}\left(\tilde{x}_{+}\right)$one has

$$
\begin{gathered}
\mathscr{W}\left(w_{+}\left(., h, E ; x_{0}, x_{+}\right), w_{+}\left(., h, E ; x_{1}, \tilde{x}_{+}\right)\right)(x)= \\
\frac{2}{h} \mathrm{e}^{\left(z_{0}(x)+z_{1}(x)\right) / h}\left\{W_{+}^{\text {even }}\left(z_{0}(x), h, z_{+}\right) W_{+}^{\text {odd }}\left(z_{1}(x), h, \tilde{z}_{+}\right)-\right. \\
\left.W_{+}^{\text {odd }}\left(z_{0}(x), h, z_{+}\right) W_{+}^{\text {even }}\left(z_{1}(x), h, \tilde{z}_{+}\right)\right\} .
\end{gathered}
$$


Notice that this proposition gives an equivalent for the wronskian of two solutions of different type (a $w_{+}$and a $w_{-}$) which is of the form $\mathrm{e}^{A / h}(1+O(h))$. In the case of two solutions of the same type (a $w_{+}$and a $\tilde{w}_{+}$), we only have an upper bound for their wronskians, which reads $O\left(h \mathrm{e}^{A / h}\right)$ for some constant $A$.

We end this section with a remark concerning the case where $E$ depends on $h$. Our constructions for the solutions are still valid, but the functions $W^{\text {odd }}$ and $W^{\text {even }}$ may not be CAS in $h$ anymore. In particular one may have (see [Ge-Gr], Prop. 2.4)

$$
\mathscr{W}\left(w_{+}, w_{-}\right)=1+O\left(h /\left(E-V_{0}\right)\right)
$$

so that Proposition 3 above is worthless when $E-V_{0}=O(h)$.

\section{Jost Solutions and Their Asymptotic Expansion as $h \rightarrow 0$}

We construct here the Jost solutions copying the procedure described in Sect. 2, the new point here being that the solutions we seek are normalized at infinity. In all this section we will work in two unbounded, simply-connected domains $\Omega^{l}(E)$ and $\Omega^{r}(E)$, where $|V(x)|<E$ and which coincide with $\mathscr{S}$ for $-\operatorname{Re} x$ and $\operatorname{Re} x$ sufficiently large respectively. The existence of such domains is of course an easy consequence of the behavior of $V$ at infinity in $\mathscr{S}$ (see assumption (A)). In these two sets we choose the determination of $t \mapsto(V(t)-E)^{1 / 4}$ which belongs to $\mathrm{e}^{2 \pi / 4} \mathbb{R}^{+}$for real $t$ so that $t \mapsto(V(t)-E)^{1 / 2}$ belongs to $i \mathbb{R}^{+}$for real $t$. To obtain the correct behavior for the phase we will use the obvious identity

$$
(V(t)-E)^{1 / 2}-i E^{1 / 2}=\frac{V(t)}{(V(t)-E)^{1 / 2}+i E^{1 / 2}}
$$

and define, with the determination chosen above

$$
z^{r, l}(x, E)=i \sqrt{E} x+\int_{ \pm \infty}^{x} \frac{V(t)}{(V(t)-E)^{1 / 2}+i \sqrt{E}} d t .
$$

For real $x$ in $\Omega^{r, l}(E)$, we shall also write this definition as

$$
z^{r, l}(x, E)=i \sqrt{E} x+i \int_{ \pm \infty}^{x}\{\sqrt{|V(t)-E|}-\sqrt{E}\} d t
$$

which shows that $z^{r, l}(x, E)$ is then purely imaginary. Notice that $z^{r, l}(x, E)$ is a primitive of $(V(t)-E)^{1 / 2}$ and that we have for any $x_{0}^{r, l} \in \Omega^{r, l}(E)$,

$$
z^{r, l}(x, E)=z\left(x, x_{0}^{r, l}, E\right)+z^{r, l}\left(x_{0}^{r, l}, E\right) .
$$

To make the coming ideas clearer, we also introduce the following

Definition 3. Let $x$ be a point in $\Omega^{l, r}(E)$. The set of points $y$ in $\Omega^{l, r}(E)$ for which $\operatorname{Re} z^{l, r}(x, E)=\operatorname{Re} z^{l, r}(y, E)$ is called the Stokes line passing through $x$.

The point is that the condition for a path to be canonical (see Definition 2) is of course that it intersects transversally the Stokes line it meets. Thanks to the absence of turning point or singularity of $V$, the local structure of the Stokes line in $\Omega^{l, r}(E)$ is particularly simple as shown by the 
Lemma 1. Let $D$ be a bounded, simply-connected domain in $\Omega^{l, r}(E)$. The function $x \mapsto z^{l, r}$ is an analytic diffeomorphism from $D$ to $z(D)$ that maps the Stokes lines in $D$ onto the vertical lines $\operatorname{Re} \xi=C^{s t}$.

Proof. The only non-trivial part is to show injectivity of the functions $z^{l, r}$. For $x \neq y$ one has with Eq. (14), using $|V(t)|<E$ in $\Omega^{l, r}(E)$,

$$
\left|z^{l, r}(x, E)-z^{l, r}(y, E)-i \sqrt{E}(x-y)\right|<\sqrt{E}|x-y|,
$$

which shows that $z(x)=z(y)$ only for $x=y$.

We consider now the global situation. With our choice for $z^{l, r}$ and the behavior of $V$ at infinity in $\mathscr{S}$ we have immediately the following

Lemma 2. The Stokes lines in $\Omega^{l, r}(E)$ are asymptotic to the horizontal lines $\operatorname{Im} x=$ $C^{s t}$. Moreover for $|x|$ big enough, the function $\operatorname{Re} z(x)$ is increasing as $\operatorname{Im} x$ decreases, and $\operatorname{Im} z(x)$ increases as $\operatorname{Re} x$ does.

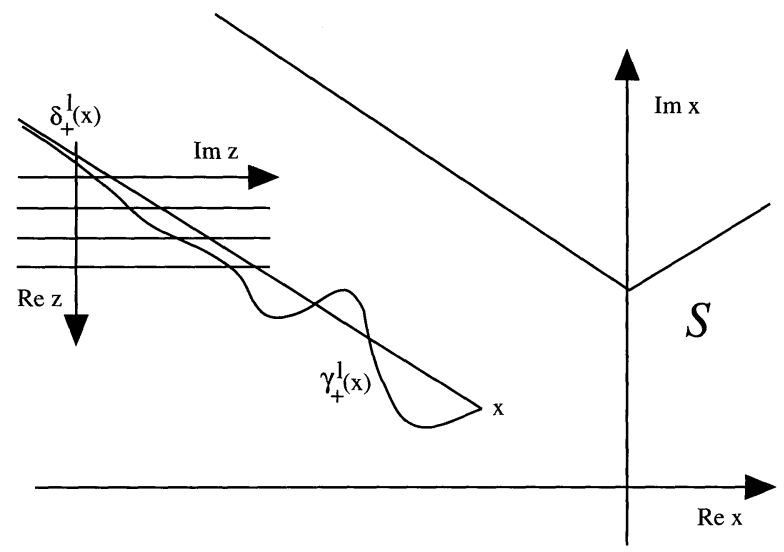

Fig. 3. Integration paths in the $z$-plane

With these two lemmas, one sees that for all $x$ in $\Omega^{l}(E)$, there exists an infinite path ending at $x, \gamma_{ \pm}^{l}(x)$, parameterized on $t \in[0,+\infty[$, asymptotic to the line $\operatorname{Im} \xi=$ $\mp \delta \operatorname{Re} \xi$ as $\operatorname{Re} \xi \rightarrow-\infty$ and such that $\xi \mapsto \pm \operatorname{Re} z(\xi)$ is strictly increasing along it (see Fig. 3). For $x$ in $\Omega^{r}(E)$ we define the same way the paths $\gamma_{ \pm}^{r}(x)$ which are asymptotic to the $\operatorname{lines} \operatorname{Im} \xi= \pm \delta \operatorname{Re} \xi$ as $\operatorname{Re} \xi \rightarrow+\infty$.

We also denote by $\Gamma_{ \pm}^{l, r}(z)$ the infinite oriented paths $z^{l, r}\left(\gamma_{ \pm}^{l, r}(x)\right)$ ending at $z^{l, r}(x, E)$, and remark that $\Gamma_{+}^{l}(z)$ and $\Gamma_{-}^{r}(z)$ are asymptotic to the line $\operatorname{Im} \zeta=\frac{1}{\delta} \operatorname{Re} \zeta$ as $\operatorname{Re} \zeta \rightarrow \mp \infty$ respectively, and that $\Gamma_{-}^{l}(z)$ and $\Gamma_{+}^{r}(z)$ are asymptotic to the line $\operatorname{Im} \zeta=-\frac{1}{\delta} \operatorname{Re} \zeta$ as $\operatorname{Re} \zeta \rightarrow \pm \infty$ respectively.

We define now four particular WKB solutions of the Schrödinger equation in $\Omega^{r}(E)$ and $\Omega^{l}(E)$. We will use Eq. (8) and Eq. (9) where the integrals are now performed along the paths $\Gamma_{ \pm}^{l, r}(z)$.

Proposition 4. Let $\Gamma_{ \pm}^{l, r}(z)$ be the path defined above. The system of recurrence equations 


$$
\left\{\begin{aligned}
\tilde{W}_{0, \pm}^{l, r}(z, h) & =1 \\
\tilde{W}_{2 p+1, \pm}^{l, r}(z, h) & =-\int_{\Gamma_{ \pm}^{l, r}(z)} \mathrm{e}^{ \pm 2(\zeta-z) / h} H(\zeta) \tilde{W}_{2 p, \pm}^{l, r}(\zeta) d \zeta \\
\tilde{W}_{2 p, \pm}^{l, r}(z, h) & =-\int_{\Gamma_{ \pm}^{l, r}(z)}^{l,} H(\zeta) \tilde{W}_{2 p-1, \pm}^{l, r}(\zeta) d \zeta
\end{aligned}\right.
$$

define a sequence of classical analytic symbols of order $\left[\frac{n+1}{2}\right]$ in $\Omega^{l, r}(E)$. Their sum

$$
\tilde{W}_{ \pm}^{l, r}(x, h, E)=\sum_{n \geq 0} \tilde{W}_{n, \pm}^{l, r}\left(z^{l, r}(x, E), h\right)
$$

is an analytic function in this set. Moreover the functions

$$
\tilde{W}_{\text {even, }}^{l, r}(x, h, E)=\sum_{n \geq 0} \tilde{W}_{2 n, \pm}^{l, r}\left(z^{l, r}(x, E), h\right)
$$

and

$$
\tilde{W}_{o d d, \pm}^{l, r}(x, h, E)=\sum_{n \geq 0} \tilde{W}_{2 n+1, \pm}^{l, r}\left(z^{l, r}(x, E), h\right)
$$

are classical analytic symbols in $\Omega^{l, r}(E)$ of order 0 and 1 respectively.

Proof. We write the proof only for $\tilde{W}_{+}^{l}$, the other cases could be treated along the same lines. We first prove convergence of the integrals above by induction. Suppose $\tilde{W}_{2 n,+}^{l}$ is bounded and analytic in $\Omega^{l}(E)$. Using Cauchy's formula and the direction of the path $\Gamma_{+}^{l}(z)$ at infinity we get

$$
\tilde{W}_{2 p+1,+}^{l}(z, h)=-\int_{\Delta_{+}^{l}(z)} \mathrm{e}^{2(\zeta-z) / h} H(\zeta) \tilde{W}_{2 p,+}^{l}(\zeta) d \zeta,
$$

where the integration is now performed along the straight line $\Delta_{+}^{l}(z)$ ending at $z$ given by $\operatorname{Im}(\zeta-z)=\frac{1}{\delta} \operatorname{Re}(\zeta-z)$. We obtain

$$
\tilde{W}_{2 p+1,+}^{l}(z, h)=-\int_{0}^{+\infty} \mathrm{e}^{-2\left(1+\frac{i}{\delta}\right) u / h}\left(H \tilde{W}_{2 p,+}^{l}\right)\left(z-\left(1+\frac{i}{\delta}\right) u\right)\left(1+\frac{i}{\delta}\right) d u,
$$

where $u=-\operatorname{Re}(\zeta-z)$, and this shows uniform convergence of the integral defining $\tilde{W}_{2 p+1,+}^{l}$ using the definition of $H$ and the behavior of $V^{\prime}$ at infinity in $\mathscr{S}$ given by assumption (A) and Cauchy's inequalities. This expression also shows that $\tilde{W}_{2 p+1,+}^{l}$ is a bounded and analytic function in $\Omega^{l}(E)$. Now we have

$$
\tilde{W}_{2 p+2,+}^{l}(z, h)=-\int_{\Gamma_{+}^{l}(z)} H(\zeta) \tilde{W}_{2 p+1,+}^{l}(\zeta) d \zeta
$$

where $H$ is obviously in $L^{1}\left(\Gamma_{+}^{l}(z)\right)$ and this answers the question of convergence of the above integrals and definition of the $\tilde{W}_{n,+}^{l}$. We look now for asymptotic expansions of these functions. We perform the change of variables $s=u / h$ in Eq. (17) and get

$$
\tilde{W}_{2 p+1,+}^{l}(z, h)=-\int_{0}^{+\infty} \mathrm{e}^{-2\left(1+\frac{\imath}{\delta}\right) s}\left(H \tilde{W}_{2 p,+}^{l}\right)\left(z-\left(1+\frac{i}{\delta}\right) h s\right)\left(1+\frac{i}{\delta}\right) h d s .
$$


Using Taylor expansion for $H \tilde{W}_{2 p,+}^{l}$ at $z$ we obtain, for any $N \in \mathbb{N}$,

$$
\tilde{W}_{2 p+1,+}^{l}(z, h)=\sum_{j=0}^{N} \frac{\partial_{z}^{j}\left(H \tilde{W}_{2 p,+}^{l}\right)(z)}{j !} \int_{0}^{+\infty} \mathrm{e}^{-2\left(1+\frac{\imath}{\delta}\right) s}\left[\left(1+\frac{i}{\delta}\right) h\right]^{j+1} s^{j} d s+O\left(h^{N+2}\right),
$$

and a direct computation gives

$$
\tilde{W}_{2 p+1,+}^{l}(z, h)=\sum_{j=0}^{N} \partial_{z}^{j}\left(H \tilde{W}_{2 p,+}^{l}\right)(z)\left(-\frac{1}{2}\right)^{j+1} h^{j+1}+O\left(h^{N+2}\right) .
$$

This shows immediately that $\tilde{W}_{2 p+1,+}^{l}(z, h)$ is a classical analytic symbol of order $p+1$ when $\tilde{W}_{2 p,+}^{l}$ is a classical analytic symbol of order $p$. Using Eq. (18), we see that $\tilde{W}_{2 p+2,+}^{l}(z, h)$ is then also a CAS of order $p+1$.

The last thing to prove is the convergence of the sum $\sum_{n \geq 0} \tilde{W}_{n,+}^{l}$. We recall the following easy result (see [Gr], Lemma 3.2)

Lemma 3. Suppose $f$ is a function in $L^{2}(] 0,+\infty[)$ and define, for all $n \geq 1$,

$$
I_{2 n}(f)=\int_{+\infty>s_{1}>s_{2}>\ldots>s_{2 n}>0}^{\mathrm{e}^{-2(1+\imath / \delta)\left(s_{1}-s_{2}+\ldots-s_{2 n}\right) / h}} f\left(s_{1}\right) f\left(s_{2}\right) \ldots f\left(s_{2 n}\right) d s_{1} d s_{2} \ldots d s_{2 n}
$$

and

$$
I_{2 n-1}(f)=\int_{+\infty>s_{1}>s_{2}>\ldots>s_{2 n-1}>0} \mathrm{e}^{-2(1+i / \delta)\left(s_{1}-s_{2}+\ldots+s_{2 n-1}\right) / h} f\left(s_{1}\right) \ldots f\left(s_{2 n-1}\right) d s_{1} d s_{2} \ldots d s_{2 n-1} .
$$

Then we have

$$
\left|I_{n}(f)\right| \leq\left(\frac{h}{2}\right)^{[(n+1) / 2]}\|f\|_{L^{2}}^{n} .
$$

This lemma and the expressions Eq. (9) and Eq. (8) for the $\tilde{W}_{n,+}^{l}$, where the path of integration is now $\Gamma_{+}^{l}(z)$, gives uniform convergence of the series, noticing that $H$ is in $L^{2}\left(\Gamma_{+}^{l}(z)\right)$ thanks to the hypothesis (A) on $V$.

We write now the corresponding WKB solutions as

$$
\tilde{w}_{ \pm}^{l, r}(x, E, h)=(V(x)-E)^{-1 / 4} \mathrm{e}^{ \pm z^{l, r}(x, E) / h} \tilde{W}_{ \pm}^{l, r}(x, h, E),
$$

and we see immediately, using uniform convergence of the integral in Eq. (17) and Eq. (18), that for $n \neq 0$,

$$
\lim _{x \rightarrow \pm \infty} \tilde{W}_{n, \pm}^{l, r}(x, h, E)=0
$$

so that we have immediately the

Proposition 5. The functions $\tilde{w}_{ \pm}^{l, r}$ are solutions of the Schrödinger equation Eq. (4) in $\Omega^{l, r}(E)$ respectively, and we have

$$
\left\{\begin{array}{l}
\lim _{x \rightarrow-\infty} \mathrm{e}^{\mp i \sqrt{E} x / h} \tilde{w}_{ \pm}^{l}(x)=(-E)^{-1 / 4} \\
\lim _{x \rightarrow+\infty} \mathrm{e}^{\mp \imath \sqrt{E} x / h} \tilde{w}_{ \pm}^{r}(x)=(-E)^{-1 / 4}
\end{array}\right.
$$

Moreover $\left(\tilde{w}_{+}^{l}, \tilde{w}_{-}^{l}\right)\left(\right.$ resp. $\left.\left(\tilde{w}_{+}^{r}, \tilde{w}_{-}^{r}\right)\right)$ is a basis of the space of solutions of Eq. (4) in $\Omega^{l}(E)\left(\right.$ resp. $\left.\Omega^{r}(E)\right)$. 
This last proposition shows that the $\tilde{w}_{ \pm}^{l, r}$ are proportional (with the same coefficient $\left.(-E)^{-1 / 4}\right)$ to the Jost solutions and that the scattering matrix $\mathbb{S}(E, h)$ can be computed in the corresponding two basis, provided these solutions are defined in a shared domain. However it will be convenient in the sequel to work with other choices for the normalization of the phase. All we have written in Sect. 3 can still be read with

$$
z\left(x, x_{0}^{l, r}, E\right)=\int_{x_{0}^{l, r}}^{x}(V(t)-E)^{1 / 2} d t
$$

instead of $z^{l, r}$, where $x_{0}^{l, r}$ are fixed points in $\Omega^{l, r}(E)$. We shall write the corresponding solutions as

$$
w_{ \pm}^{l, r}\left(x, E, h ; x_{0}^{l, r}\right)=(V(x)-E)^{-1 / 4} \mathrm{e}^{ \pm z\left(x, x_{0}^{l, r}, E\right) / h} W_{ \pm}^{l, r}\left(x, h, E, x_{0}^{l, r}\right)
$$

and we have the

Proposition 6. The functions $w_{ \pm}^{l, r}$ are solutions of the Schrödinger equation (4) in $\Omega^{l, r}(E)$ respectively, and we have

$$
\tilde{w}_{ \pm}^{l, r}(x, E, h)=\mathrm{e}^{ \pm z^{l, r}\left(x_{0}^{l, r}, E\right)} w_{ \pm}^{l, r}\left(x, E, h ; x_{0}^{l, r}\right) .
$$

Proof. We compute the wronskian of $w_{+}^{l}$ and $\tilde{w}_{+}^{l}$ at $x$ in $\Omega^{l}(E)$ using Eq. (10). Then letting $x \rightarrow-\infty$ and considering Eq. (21) which is true both for $w$ and $\tilde{w}$, we see that this wronskian is 0 , so that these solutions are proportional. But we have

$$
\lim _{x \rightarrow-\infty} \frac{\tilde{w}_{+}^{l}(x, E, h)}{\mathrm{e}^{\imath \sqrt{E} x / h}}=(-E)^{-1 / 4}
$$

and, with Eq. (16),

$$
\lim _{x \rightarrow-\infty} \frac{\mathrm{e}^{z^{l}\left(x_{0}^{l}, E\right)} w_{+}^{l}\left(x, E, h ; x_{0}^{l}\right)}{\mathrm{e}^{i \sqrt{E} x / h}}=(-E)^{-1 / 4} .
$$

The same arguments lead to the three other equalities.

We end this section with a remark concerning the so-called long range case, where $V$ goes to zero at infinity like $|x|^{-r}$ with $0<r \leq 1$. Then, as shown by Eq. (14) the primitives of $(V-E)^{1 / 2}$ do no longer behave at infinity like $i \sqrt{E} x+C^{s t}$, so one has to define Jost solutions another way. The good one (see for instance [Ag-Kl]) is to consider the solutions which behave at infinity like $\mathrm{e}^{ \pm z\left(x, E, x_{0}\right) / h}$ for some natural choice of $x_{0}$, and there is no problem to adapt what we are doing in Sect. 4 below and obtain the corresponding results in the long range case.

\section{Scattering for Energies Far Away From the Extrema of the Potential}

We shall use in this section the notations introduced in Part 3 and compute some of the elements of the scattering matrix $\mathbb{S}(E, h)$ that we may write as

$$
s_{11}(E, h)=\frac{\mathscr{W}\left(\tilde{w}_{+}^{l}, \tilde{w}_{-}^{l}\right)}{\mathscr{W}\left(\tilde{w}_{+}^{r}, \tilde{w}_{-}^{l}\right)} \quad \text { and } \quad s_{21}(E, h)=\frac{\mathscr{W}\left(\tilde{w}_{+}^{l}, \tilde{w}_{+}^{r}\right)}{\mathscr{W}\left(\tilde{w}_{-}^{l}, \tilde{w}_{+}^{r}\right)} .
$$


As mentioned at the end of Sect. 3 it will be more convenient to work with other solutions than the $\tilde{w}$. Denoting by $w_{ \pm}^{l, r}\left(x, E, h ; x_{0}^{l, r}\right)$ the ones defined by Eq. (22), we get easily with Proposition 6 the following useful formulas:

$$
s_{11}(E, h)=\mathrm{e}^{\left(z^{l}\left(x_{0}^{l}, E\right)-z^{r}\left(x_{0}^{r}, E\right)\right) / h} \frac{\mathscr{W}\left(w_{+}^{l}, w_{-}^{l}\right)}{\mathscr{W}\left(w_{+}^{r}, w_{-}^{l}\right)}
$$

and

$$
s_{21}(E, h)=-\mathrm{e}^{2 z^{l}\left(x_{0}^{l}, E\right) / h} \frac{\mathscr{W}\left(w_{+}^{l}, w_{+}^{r}\right)}{\mathscr{W}\left(w_{+}^{r}, w_{-}^{l}\right)} .
$$

These expressions are of course formal in the sense that they may involve solutions which are not defined in a shared domain. We shall now extend these functions in order that Eq. (23) and Eq. (24) make sense.

\subsection{Transmission through a barrier}

We suppose here that $V$ satisfies assumption (A) and that $V_{m}-C>E>C$ for a constant $C>0$ independent of $h$, where $V_{m}$ is the lowest local extremum of $V$ on the real axis (see Fig. 1). There exists then only two real turning points $a(E)<b(E)$, and we suppose that they are simple. In that case the Stokes lines are as shown in Fig. 4.

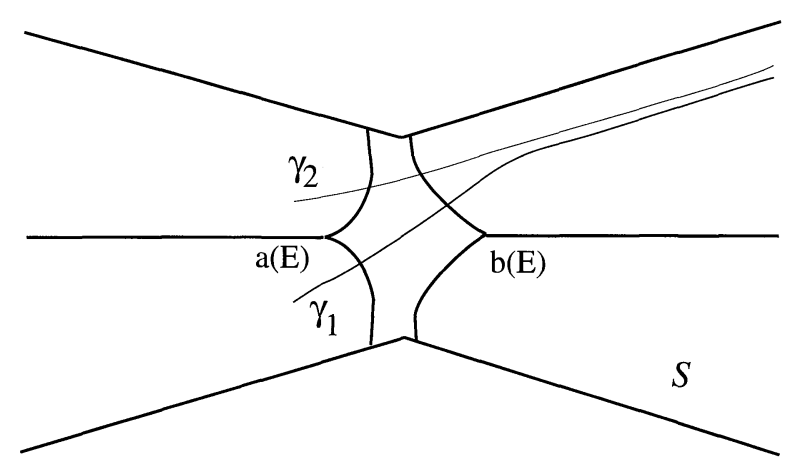

Fig. 4. Continuations of the solutions in $\mathscr{S}$ : real turning points

We first compute $s_{11}(E, h)$ using Eq. (23). We recall that

$$
\mathscr{W}\left(\tilde{w}_{+}^{l}, \tilde{w}_{-}^{l}\right)=\frac{2}{h}
$$

so the only remaining quantity is the wronskian of $\tilde{w}_{+}^{r}$ and $\tilde{w}_{-}^{l}$ or equivalently $W\left(w_{+}^{r}, w_{-}^{l}\right)$. We have to extend the function $w_{+}^{r}$, which is defined in $\Omega^{r}(E)$, into $\Omega^{l}(E)$. We recall that we have chosen the determination of $t \mapsto(V(t)-E)^{1 / 4}$ which belongs to $\mathrm{e}^{i \pi / 4} \mathbb{R}^{+}$for real $t$ in $\Omega^{r}(E)$. Then we define the function $z$ in $\Omega^{r}(E)$, by

$$
z(x, b(E), E)=\int_{b(E)}^{x}(V(t)-E)^{1 / 2} d t .
$$


Thanks to the structure of the Stokes lines between $a(E)$ and $b(E)$, we can find a path $\gamma_{1}$ from $+\infty(1+i \delta)$ to $-\infty(1+i \delta)$ transverse to the Stokes lines, cutting the real axis at $x=0$ and along which we can propagate this determination for $(V(t)-E)^{1 / 4}$ (see Fig. 4). The determination we obtain that way in $\Omega^{l}(E)$ is such that $(V(t)-E)^{1 / 4}$ belongs again to $\mathrm{e}^{i \pi / 4} \mathbb{R}^{+}$for real $t$. Notice that between $a(E)$ and $b(E)$ on the real axis, where $V(t)>E$, we have

$$
(V(t)-E)^{1 / 2}=-\sqrt{V(t)-E} .
$$

We shall denote by ${ }^{1} w_{+}^{r}$ the extension of $w_{+}^{r}(x, E, h ; b(E))$ along $\gamma_{1}$ and compute the wronskian of the solutions ${ }^{1} w_{+}^{r}$ and $w_{-}^{l}=w_{-}^{l}(x, E, h ; a(E))$ at $y$. With Eq. (11) we get

$$
\mathscr{W}\left({ }^{1} w_{+}^{r}, w_{-}^{l}\right)(y)=\frac{2}{h} \mathrm{e}^{(z(y, b(E), E)-z(y, a(E), E)) / h}(1+h \psi(h))
$$

and with Eq. (25)

$$
\mathscr{W}\left(w_{+}^{r}, w_{-}^{l}\right)=\frac{2}{h} \mathrm{e}^{S(E) / h}(1+h \psi(h)),
$$

where $\psi(h)$ is a classical analytic symbol of positive order. Equation (23) gives

$$
s_{11}(E, h)=\left(1+h \phi_{1}(h)\right) \exp \left\{-\frac{S(E)}{h}\right\} \exp \left\{\frac{i}{h} S_{\infty}(E)\right\},
$$

where $\phi_{1}$ again is a CAS of non-negative order, and where we have written

$$
S_{\infty}(E)=z^{l}(a(E), E)-z^{r}(b(E), E)
$$

that is precisely

$$
S_{\infty}(E)=-\sqrt{E}(b(E)-a(E))+\int_{b(E)}^{+\infty} Q(t, E) d t+\int_{-\infty}^{a(E)} Q(t, E) d t
$$

with $Q(t, E)=\sqrt{E-V(t)}-\sqrt{E}$.

Let's compute now $s_{21}$ with Eq. (24), where only $\mathscr{W}\left(w_{+}^{l}, w_{+}^{r}\right)$ is not known. If we used the same extension of $w_{+}^{r}$ into $\Omega^{l}(E)$ as for the computation of $s_{11}$, we would only obtain an upper bound for $s_{21}$ (see discussion after Proposition 3). Instead we choose a point $y \in \Omega^{l}(E)$ and a canonical path $\gamma_{2}(y)$ going from $+\infty(1+i \delta)$ to $y$, which stays above the turning points (see Fig. 4), and we extend the solution $w_{+}^{r}$ along $\gamma_{2}$. If we denote this extension by ${ }^{2} w_{+}^{r}$, we obtain in $\Omega^{l}(E)$ :

$$
{ }^{2} w_{+}^{r}(y, E, h ; b(E))=-i \mathrm{e}^{S(E) / h}(V(y)-E)^{-1 / 4} \mathrm{e}^{-z(y, a(E), E) / h} \sum_{n \geq 0}{ }^{2} W_{n,+}^{r}(y),
$$

where the determination of $(V(y)-E)^{1 / 4}$ is the one we have fixed at the beginning of this section, and we see that ${ }^{2} w_{+}^{r}$ is of the type $-i \mathrm{e}^{S(E) / h} w_{-}$for a solution $w_{-}$ defined in $\Omega^{l}(E)$.

Now we compute $\mathscr{W}\left(w_{+}^{l},{ }^{2} w_{+}^{r}\right)$ at $y$ and obtain, for a CAS $\chi(h)$ of non-negative order

$$
\mathscr{W}\left(w_{+}^{l},{ }^{2} w_{+}^{r}\right)(y)=-i \mathrm{e}^{S(E) / h} \mathscr{W}\left(w_{+}^{l}, w_{-}\right)(y)=-\frac{2 i}{h} \mathrm{e}^{S(E) / h}(1+\chi(h))
$$

so that, with Eq. (26),

$$
s_{21}(E, h)=\mathrm{ie}^{2 z^{l}(a(E), E) / h}\left(1+h \phi_{2}(h)\right) .
$$

This ends the proof of Theorem 1. 


\subsection{Over-barrier reflection}

We suppose now that the energy level $E$ is above the maximum value $V_{0}$ of the potential $V$ on the real axis. More precisely we have to suppose here that $E-V_{0}>$ $C>0$ for a constant $C$ independent of $h$. If we did not make any further assumption on $V$, we could take the same domain for $\Omega^{l}(E)$ and $\Omega^{r}(E)$ and compute the different wronskians in that set. As we have already noticed above, we would obtain that way an upper bound for the reflection coefficient. Because we want to compare the results of this section with the one of Sect. 6, we prefer to suppose that for these $E$, the domain $\mathscr{S}$ contains two complex-conjugate simple turning points and we denote them by $a(E)$ and $b(E)$ with $\operatorname{Im} a(E)<0<\operatorname{Im} b(E)$. We also have to state a geometrical hypothesis on the structure of the Stokes lines, which should be as in Fig. 5. Notice first that $x \mapsto z(x, 0)$ is an analytical diffeomorphism from the real $x$-axis to the imaginary $z$-axis. The function $z^{-1}$ extends to a strip in the $z$-plane whose image by $z^{-1}$ is bounded by two Stokes lines. Our assumption is that each of these Stokes lines contains no turning points but exactly one among $a(E)$ and $b(E)$. Notice that this is true when $E$ is close enough to a quadratic maximum of $V$.

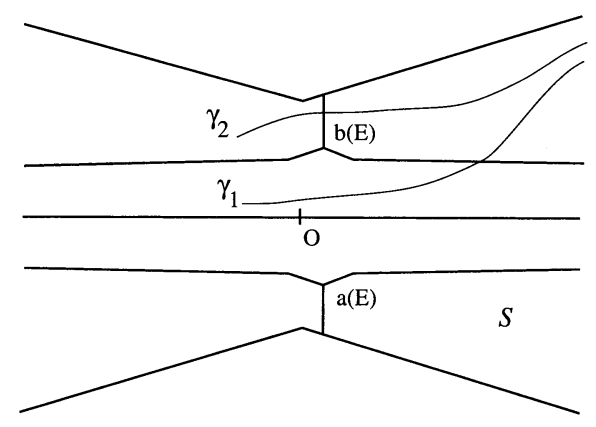

Fig. 5. Continuations of the solutions in $\mathscr{S}$ : two complex turning points

In $\Omega^{l}(E)$, which is also $\Omega^{r}(E)$, we choose the determination of $(V(x)-E)^{1 / 4}$ which belongs to $\mathrm{e}^{i \pi / 4} \mathbb{R}^{+}$for real $x$ and we define as in Sect. 3 the two solutions $w_{-}^{l}(x)=w_{-}^{l}(x, E, h ; a(E))$ and $w_{+}^{r}(x)=w_{+}^{r}(x, E, h ; b(E))$. Notice that, with the same notations as in Sect. 4.1, $w_{+}^{r}(x)$ can be written as ${ }^{1} w_{+}^{r}(x)$ for a path $\gamma_{1}$ which stays in $\Omega^{l}(E)$ (see Fig. 5). Equation (11) gives first

$$
\mathscr{W}\left(w_{+}^{r}, w_{-}^{l}\right)=\frac{2}{h} \exp \left\{-\frac{1}{h} \int_{a(E)}^{b(E)}(V(t)-E)^{1 / 2} d t\right\}\left(1+h^{2} \psi_{1}(h)\right),
$$

where $\psi_{1}(h)$ is a CAS of non-negative order. We use then Eq. (23) and get, for another CAS $\phi_{1}$,

$$
s_{11}=\left(1+h^{2} \phi_{1}(h)\right) \exp \left\{\frac{1}{h}\left(z^{l}(a(E), E)-z^{r}(b(E), E)+\int_{a(E)}^{b(E)}(V(t)-E)^{1 / 2} d t\right)\right\}
$$

that is finally, with $Q(t, E)=i \sqrt{E-V(t)}-i \sqrt{E}$ for real $t$,

$$
s_{11}=\left(1+h^{2} \phi_{1}(h)\right) \exp \left\{\frac{1}{h} \int_{-\infty}^{+\infty} Q(t, E) d t\right\} .
$$


Next we have to compute $\mathscr{W}\left(w_{+}^{l}, w_{+}^{r}\right)$. We use the same idea as in Sect. 4.1 and extend the solution $w_{+}^{r}$ along a path $\gamma_{2}$ which comes from $+\infty(1+i \delta)$ and stays above the turning points (see Fig. 5). The corresponding solution is denoted by ${ }^{2} w_{+}^{r}$ and we have

$$
{ }^{2} w_{+}^{r}(y)=-i(V(y)-E)^{-1 / 4} \mathrm{e}^{-z(y, b(E), E) / h} \sum_{n \geq 0}{ }^{1} W_{n,+}^{r}(y),
$$

where the determination of $(V(y)-E)^{1 / 4}$ is the one we have chosen above. Again with Eq. (11) we get

$$
\mathscr{W}\left(w_{+}^{l},{ }^{2} w_{+}^{r}\right)=-\frac{2 i}{h}\left(1+h^{2} \psi_{2}(h)\right) \exp \left\{\frac{1}{h} \int_{a(E)}^{b(E)}(V(t)-E)^{1 / 2} d t\right\},
$$

and with Eq. (24)

$$
s_{21}=i\left(1+h^{2} \phi_{2}(h)\right) \exp \left\{\frac{2}{h}\left(\int_{a(E)}^{b(E)}(V(t)-E)^{1 / 2} d t+z^{l}(a(E), E)\right)\right\}
$$

so that at last

$$
s_{21}=i\left(1+h^{2} \phi_{2}(h)\right) \exp \left\{\frac{2}{h}\left(\int_{-\infty}^{0} Q(t, E) d t+\int_{0}^{b(E)}(V(t)-E)^{1 / 2} d t\right\} .\right.
$$

We have proven the following

Proposition 7. Suppose that $V$ satisfies assumption (A). We also suppose that $E>V_{0}$ is fixed and that the hypothesis at the beginning of this section are satisfied for this $E$.

Then we have

$$
\left\{\begin{array}{l}
s_{11}=\left(1+h^{2} \phi_{1}(h)\right) \exp \left\{\frac{1}{h} \int_{-\infty}^{+\infty} Q(t, E) d t\right\} \\
s_{21}=i\left(1+h^{2} \phi_{2}(h)\right) \exp \left\{\frac{2}{h}\left(\int_{-\infty}^{0} Q(t, E) d t+\int_{0}^{b(E)}(V(t)-E)^{1 / 2} d t\right\} .\right.
\end{array}\right.
$$

The reader may notice that these formula are precisely those of Theorem 3 .

\section{Microlocal Study Near a Quadratic Maximum}

In this section we study precisely what happens when the energy $E$ is very close to the maximum $V_{0}$ of the potential $V$ on the real axis, under the assumption that this maximum is of quadratic type. We suppose $V$ satisfies assumptions (A) and (B), which permits us to control the location and the nature of the turning points. We recall that there exists a constant $C>0$ depending only on the potential $V$ such that if $\left|V_{0}-E\right|<C$, there exist in a complex neighborhood of 0 exactly two simple turning points which are real and of opposite sign for $E<V_{0}$ and complex-conjugate for $E>V_{0}$, close to $\pm \frac{1}{k} \sqrt{2\left(V_{0}-E\right)}$. The difficulties come from the fact that these two turning points coalesce when $E$ goes to $V_{0}$ as $h$ vanishes. 


\subsection{Reduction to a normal form}

We use here as in [Mä] and [Ra] some techniques which have been introduced by J.Sjöstrand and B.Helffer (see [He-Sj]) and that we have briefly described in the appendix. The initial remark is that the Schrödinger equation (4) may be written as

$$
P(x, \hbar D) u=\mu u
$$

with $P(x, \hbar D)=\frac{\hbar^{2}}{2} D^{2}+V_{0}(x), V_{0}(x)=\frac{V(x)-V_{0}}{k^{2}}$ and $\mu=\frac{E-V_{0}}{k^{2}}$. Then we notice that the $\hbar$-symbol of this operator is close to the symbol associated to Weber's equation $\left(\xi^{2}-x^{2}\right) / 2$ for $x$ close to 0 , and is mapped into a symbol close to $q(x, \xi)=x \xi$ by the rotation by $\pi / 4$. The following theorem (see [He-Sj] App. b) allows us to work microlocally in that direction:

Proposition 8. (B.Helffer, J.Sjöstrand) There exists a real-analytic canonical transformation $\kappa$, defined in a neighborhood of $(0,0)$ with values in a real neighborhood of $(0,0)$ and a real-analytic function $f_{0}$ defined near 0 such that:

1. $f_{0}(0)=0, f_{0}^{\prime}(0)=1$.

2. $\kappa(0,0)=(0,0), d \kappa(0,0)=\kappa_{\pi / 4}$, where $\kappa_{\pi / 4}$ is the rotation by $\pi / 4$ around $(0,0)$ in $T^{*} \mathbb{R}$.

3. $f_{0} \circ p \circ \kappa=q$.

Moreover there exists a unitary Fourier Integral Operator $U$, with canonical transformation $\kappa$ and a real-valued classical analytic symbol $F(t, \hbar)$ of order 0 whose principal symbol is $f_{0}$ such that:

4. $U^{*} F(P, \hbar) U=Q$ microlocally near $(0,0)$, where $Q=\frac{1}{2}(x \hbar D+\hbar D x)$.

5. $\Gamma U=U A$, where $\Gamma$ is the complex conjugation operator and $A=\Gamma \mathscr{F}_{\hbar}^{-1}=\mathscr{T}_{\hbar} \Gamma$.

In particular the point (4) of this proposition gives

Corollary 1. Let $\mu$ be a small enough real number. The equation $Q u=\mu^{\prime} u$ is microlocally in a neighborhood of $(0,0)$ equivalent to the equation $P U u=\mu U u$ with $\mu^{\prime}=F(\mu, \hbar)$.

In the sequel of this section, we will study more closely the F.I.O. $U$ and the symbol $F$ (see also [Mä]). Most of the following results are consequences of the symmetry imposed to $U$ by the identity (5) above. Because we are interested only on its principal symbol we write formally $U$ as

$$
U u(x)=\mathrm{e}^{\imath C / \hbar} \int \mathrm{e}^{i \psi(x, y) / \hbar} \alpha(x, y, \hbar) u(y) \frac{d y}{\sqrt{2 \pi \hbar}},
$$

where $\alpha(x, y, \hbar)=\alpha_{0}(x, y)+\hbar \alpha_{1}(x, y)+\ldots$ is a CAS, and $\psi(x, y)$ the phase function defined in a neighborhood of $(0,0)$ by

$$
\kappa\left(y,-\partial_{y} \psi(x, y)\right)=\left(x, \partial_{x} \psi(x, y)\right)
$$

In fact we should have written

$$
U u(x, \hbar)=\iint \mathrm{e}^{i \psi(x, y, \theta) / \hbar} \alpha(x, y, \theta, \hbar) u(y, \hbar) \frac{d \theta}{\sqrt{2 \pi \hbar}} d y
$$

where the integration is performed along a good contour $\gamma(y, \vartheta)$ (cf. [Sj2], Chap.4 or [De], Chap.1), and the expression above is obtained with a stationary phase expansion. 
Proposition 9. For any $(x, y)$ near $(0,0)$ one has

1. $\psi(x, y)=\psi_{0}(x, y)+O\left((x, y)^{3}\right)$, where $\psi_{0}(x, y)=-\frac{x^{2}}{2}+\sqrt{2} x y-\frac{y^{2}}{2}$ is the phase associated to the canonical transformation $\kappa \frac{\pi}{4}$.

2. $y=\partial_{y} \psi\left(x, \partial_{y} \psi(x, y)\right)$ and $\partial_{x} \psi(x, y)=-\partial_{x} \psi^{4}\left(x, \partial_{y} \psi(x, y)\right)$.

3. We can choose $C=0$ and then $\alpha_{0}(0,0)=2^{1 / 4} \mathrm{e}^{i \pi / 8}$. Defining the CAS $\sigma=\sigma_{0}+$ $\hbar \sigma_{1}+\ldots$ by $\sigma(x, y, \hbar)=\frac{\alpha(x, y, \hbar)}{\alpha_{0}(0,0)}$, we get $\sigma_{0}(0,0)=1$ and

$$
\frac{\overline{\sigma_{0}(x, y)}}{\left|\partial_{y}^{2} \psi(x, y)\right|^{1 / 4}}=\frac{\sigma_{0}\left(x, \partial_{y} \psi(x, y)\right)}{\left|\partial_{y}^{2} \psi\left(x, \partial_{y} \psi(x, y)\right)\right|^{1 / 4}} .
$$

Proof. (1) is a direct consequence of relation (2) in Proposition 8, and computing $\psi_{0}(x, y)$ is straightforward. We examine now the second point. Point (5) of Proposition 8 gives

$$
\kappa \circ \kappa_{A}=\kappa_{\Gamma} \circ \kappa
$$

where $\kappa_{A}(x, \xi)=(-\xi,-x)$ and $\kappa_{\Gamma}(x, \xi)=(x,-\xi)$ are the canonical transformations for the F.I.O. $A$ and $\Gamma$ respectively. Thus for $(y,-\eta)$ close enough to $(0,0)$ and defining $(x, \xi)=\kappa(-\eta, y)$, we have $(x,-\xi)=\kappa(y,-\eta)$. With Eq. (28), we then get

$$
\left\{\begin{array}{l}
\xi=\partial_{x} \psi(x, \eta)=-\partial_{x} \psi(x, y) \\
y=\partial_{y} \psi(x, \eta) \\
\eta=\partial_{y} \psi(x, y)
\end{array}\right.
$$

and this gives the two identities of (2). For the last point one notices that for $a(x, h)=$ $\mathrm{e}^{-x^{2} / 2 \hbar}$ we have $A a=a$, and again with relation (5) of Proposition 8 we obtain $\Gamma U a=U a$ that is

$$
\mathrm{e}^{i C / \hbar} \int \mathrm{e}^{i \psi(x, y) / \hbar} \alpha(x, y, \hbar) \mathrm{e}^{-y^{2} / 2 \hbar} \frac{d y}{\sqrt{2 \pi \hbar}} \in \mathbb{R} .
$$

In particular for $x=0$, there exists a real number $\tilde{\alpha}_{0}$ such that

$$
\mathrm{e}^{i C / \hbar} \int \mathrm{e}^{-(1+i) y^{2}+O\left(y^{3}\right) / 2 \hbar} \alpha(0, y, \hbar) \frac{d y}{\sqrt{2 \pi \hbar}}=\tilde{\alpha}_{0}
$$

With a stationary phase expansion this gives

$$
\alpha_{0}(0,0)=2^{1 / 4} \mathrm{e}^{-i C / \hbar} \mathrm{e}^{\imath \pi / 8} \tilde{\alpha}_{0} .
$$

Last, thanks to the unitarity of $U$, we have $|U a(0)|=|a(0)|=1$ and $\tilde{\alpha}_{0}= \pm 1$. We can choose $\tilde{\alpha}_{0}=1$ without loss of generality, and this proves the first part of (3). Let's work now with $\sigma(x, y, \hbar)=\sigma_{0}(x, y)+\hbar \sigma_{1}(x, y)+\ldots$ defined by $\sigma(x, y, \hbar)=$ $2^{-1 / 4} \mathrm{e}^{-\imath \pi / 8} \alpha(x, y, \hbar)$. We do have $\sigma_{0}(0,0)=1$, and relation (5) of Proposition 8 gives

$$
\begin{aligned}
& \frac{2^{1 / 4} \mathrm{e}^{-\imath \pi / 8}}{\sqrt{2 \pi \hbar}} \int \mathrm{e}^{-i \psi(x, y) / \hbar} \overline{\sigma(x, y, \hbar)} \bar{u}(y) d y=\Gamma U u(x)= \\
& U A u(x)=\frac{2^{1 / 4} \mathrm{e}^{i \pi / 8}}{\sqrt{2 \pi \hbar}} \int \mathrm{e}^{i \psi(x, y) / \hbar} \sigma(x, y, \hbar) A u(y) d y
\end{aligned}
$$

where $A u(y)=\mathscr{T} \hbar \Gamma u(y)$. So we have 


$$
\mathrm{e}^{-i \pi / 4} \int \mathrm{e}^{-i \psi(x, y) / \hbar} \overline{\sigma(x, y, \hbar) u(y)} d y=\iint \mathrm{e}^{i(\psi(x, y)-y \eta) / \hbar} \sigma(x, y, \hbar) \overline{u(\eta)} \frac{d \eta}{\sqrt{2 \pi \hbar}} d y
$$

One performs now stationary phase expansion to get rid of integration with respect to $y$. Critical points $y_{c}(x, \eta)$ of $y \mapsto \psi(x, y)-y \eta$ are given by $\eta=\partial_{y}\left(x, y_{c}(x, \eta)\right)$ and with (2)

$$
y_{c}(x, \eta)=\partial_{\eta} \psi(x, \eta)
$$

The corresponding critical value of the phase happens to be $f_{c}(x, \eta)=-\psi(x, \eta)$ because $f_{c}(0,0)=0$ and, using again $(2)$, one has

$$
\partial_{x} f_{c}(x, \eta)=-\partial_{x} \psi(x, \eta) \quad \text { and } \quad \partial_{\eta} f_{c}(x, \eta)=-\partial_{\eta} \psi(x, \eta) .
$$

Thus Eq. (5.1) gives

$$
\int \mathrm{e}^{-i \psi(x, y) / \hbar} \overline{\sigma(x, y, \hbar) u(y)} d y=\int \mathrm{e}^{-\imath \psi(x, \eta) / \hbar} \frac{\sigma_{0}\left(x, \partial_{\eta} \psi(x, \eta)\right)+O(\hbar)}{\left|\partial_{\eta \eta}^{2} \psi\left(x, \partial_{\eta} \psi(x, \eta)\right)\right|^{1 / 2}} \overline{u(\eta)} d \eta
$$

and changing notations

$$
\overline{\sigma_{0}(x, y)}=\frac{\sigma_{0}\left(x, \partial_{y} \psi(x, y)\right)}{\left|\partial_{y y}^{2} \psi\left(x, \partial_{y} \psi(x, y)\right)\right|^{1 / 2}} .
$$

The last part of (3) is then obtained differentiating (2), that is

$$
1=\partial_{y y}^{2} \psi\left(x, \partial_{y} \psi(x, y)\right) \partial_{y y}^{2} \psi(x, y)
$$

For the symbol $F(t, \hbar)$ we have

Proposition 10. There exists a real neighborhood $\omega$ of 0 such that, for all $\mu$ in $\omega$

$$
f_{0}(\mu)=-\frac{1}{\pi} S(\mu) \quad \text { and } \quad f_{1}(\mu)=0
$$

where $S(\mu)$ is the classical action between the corresponding two turning points $a(\mu)$ and $b(\mu)$ :

$$
S(\mu)=\left\{\begin{array}{ll}
\int_{a(\mu)}^{b(\mu)} \sqrt{V_{0}(t)-\mu} d t & \text { for } \mu<0 \\
i \int_{a(\mu)}^{b(\mu)} \sqrt{|V(t)-\mu|} d t & \text { for } \mu>0
\end{array} .\right.
$$

Proof. Thanks to the definition of the functional calculus we use (see [He-Sj], App. a.3) and in particular because the symbol of $(z-P)^{-1}$ is $(z-p(x, \xi))^{-1}+O\left(\hbar^{2}\right)$, it is clear that denoting by $s$ the symbol of $F(P, \hbar)$, we have $s(x, \xi)=F(p(x, \xi))+O\left(\hbar^{2}\right)$. On the other hand, noticing that one can consider many phases for a $\hbar$-F.I.O., the difference of two of them being of order $\hbar$, it is easy to see that there exits an $\hbar$ dependent canonical transformation $\tilde{\kappa}$ associated to the F.I.O. $U$ such that identity (4) of Proposition 8 gives $s \circ \tilde{\kappa}=q+O\left(\hbar^{2}\right)$. So we get

$$
F \circ p \circ \tilde{\kappa}(x, \xi)=x \xi+O\left(\hbar^{2}\right) \text {. }
$$

But we also have

$$
f_{0} \circ p \circ \kappa=q(x, \xi)=x \xi
$$


Using these two relations and the fact that the canonical transformations $\kappa$ and $\tilde{\kappa}$ preserve oriented area, we compute in two different ways the following action integral

$$
I_{p, \mu}=\oint_{\gamma} \xi d x
$$

where the integration path is a closed complex curve $\gamma$ inside $p^{-1}(\mu)$. With Eq. (31) and denoting by $\tilde{q}$ the right-hand side of this identity, recalling also that $\mu^{\prime}=F(\mu, \hbar)$, we get

$$
I_{p, \mu}=I_{\tilde{q}, \mu^{\prime}}=\oint_{\gamma^{\prime}} \xi d x
$$

where the integration path $\gamma^{\prime}$ of $I_{\tilde{q}, \mu^{\prime}}$ is such that its image under $\tilde{\kappa}$ is $\gamma$. On the other hand Eq. (32) gives

$$
I_{p, \mu}=I_{q, \mu_{0}^{\prime}}=\oint_{\gamma_{0}^{\prime}} \xi d x,
$$

where $\mu_{0}^{\prime}=f_{0}(\mu)$ and the image by $\kappa$ of $\gamma_{0}^{\prime}$ has to be $\gamma$. Let's compute first the last of these integrals. We choose $\gamma_{0}^{\prime}$ to be the path $\left\{(x(t), \xi(t)), x(t)=r \mathrm{e}^{i t}, \xi(t)=\frac{\mu_{0}^{\prime}}{x(t)}, t \in\right.$ $[0,2 \pi]\}$ with $r$ a fixed positive real number such that the turning points $a(\mu)$ and $b(\mu)$ are inside the closed curve $\gamma_{0}^{\prime}$ for any $\mu$ small enough. Then we have

$$
I_{q, \mu_{0}^{\prime}}=2 i \pi \mu_{0}^{\prime} \text {. }
$$

When $\mu$ is small enough so is $\mu_{0}^{\prime}$ and the loop $\gamma_{0}^{\prime}$ is close to the circle $\left\{\left(r \mathrm{e}^{i t}, 0\right), t \in\right.$ $[0,2 \pi]\}$; its image $\gamma$ under $\kappa$ is thus close to $\left\{\left(r_{0} \mathrm{e}^{i t}, r_{0} \mathrm{e}^{i t}\right), t \in[0,2 \pi]\right\}$ with $r_{0}=$ $r / \sqrt{2}$. On $\gamma$ we have of course $\xi(t)=\left[2\left(\mu-V_{0}(x(t))\right]^{1 / 2}\right.$, where we have chosen the determination of $x \mapsto\left(\mu-V_{0}(x)\right)^{1 / 2}$ which is real and positive for $x=r$. Deforming the path $\gamma$, and being a little careful with the determinations of the square root as in Sect. 4, we get

$$
I_{p, \mu}=\left\{\begin{array}{ll}
-2 i \int_{a(\mu)}^{b(\mu)} \sqrt{2\left(V_{0}(x)-\mu\right)} d x & \text { for } \mu<0 \\
2 \int_{a(\mu)}^{b(\mu)} \sqrt{\left.2\left|V_{0}(x)-\mu\right|\right)} d x & \text { for } \mu>0
\end{array},\right.
$$

and this gives the value of $\mu_{0}^{\prime}$.

We compute now $I_{\tilde{q}, \mu^{\prime}}$ on a path $\tilde{\gamma}(\hbar)$ which has to be close to $\left\{\left(r \mathrm{e}^{i t}, 0\right), t \in\right.$ $[0,2 \pi]\}$. Thanks to the fact that $\tilde{q}=q+O\left(\hbar^{2}\right)$ and using analyticity in Eq. (31), we get

$$
I_{\tilde{q}, \mu^{\prime}}=2 i \pi \mu^{\prime}+O\left(h^{2}\right)
$$

and because $I_{q, \mu_{0}^{\prime}}=I_{\tilde{q}, \mu^{\prime}}$

$$
2 i \pi f_{0}(\mu)=2 i \pi F(\mu, \hbar)+O\left(\hbar^{2}\right)
$$

so $f_{1}$ is identically 0 . 


\subsection{The branching model}

We study here equation $Q u=\mu^{\prime} u$ for some real $\mu^{\prime}$. It is easy to get two maximal solutions for this order one ordinary differential equation and we can consider them as distributions in $\mathscr{S}^{\prime}(\mathbb{R})$ :

$$
u_{ \pm}^{0}\left(x, \mu^{\prime}, \hbar\right)=H( \pm x)|x|^{-1 / 2} \mathrm{e}^{\imath \mu^{\prime} \log |x| / \hbar},
$$

where $H(x)$ is the Heaviside function. Thanks to the symmetry of $Q$ with respect to $x$ and $\xi$ we have $\left[Q, \mathscr{F}_{\hbar}^{-1} \Gamma\right]=0$ and we obtain two other solutions in $\mathscr{S}^{\prime}$,

$$
v_{ \pm}^{0}\left(x, \mu^{\prime}, \hbar\right)=\mathscr{F}_{\hbar}^{-1} \Gamma u_{ \pm}^{0}\left(\xi, \mu^{\prime}, \hbar\right)=\mathscr{F}_{\hbar}^{-1}\left(H( \pm \xi)|\xi|^{-1 / 2-i \mu^{\prime} / \hbar}\right) .
$$

These four solutions are not linearly independent as proven by the

Proposition 11. Let $\mu^{\prime}$ be a real number. If $u$ in $\mathscr{D}^{\prime}$ is a solution of equation $Q u=\mu^{\prime} u$, then $u$ is in $\mathscr{S}^{\prime}$ and there exist four complex numbers $\alpha_{+}, \alpha_{-}, \beta_{+}$and $\beta_{-}$such that

$$
u=\alpha_{+} u_{+}^{0}+\alpha_{-} u_{-}^{0}=\beta_{+} v_{+}^{0}+\beta_{-} v_{-}^{0}
$$

and

$$
\left(\begin{array}{c}
\alpha_{+} \\
\alpha_{-}
\end{array}\right)=B_{\mu^{\prime}}(\hbar)\left(\begin{array}{c}
\beta_{+} \\
\beta_{-}
\end{array}\right)
$$

where the branching matrix $B_{\mu^{\prime}}(\hbar)$ is unitary and given by

$$
B_{\mu^{\prime}}(\hbar)=\frac{\hbar^{-i \mu^{\prime} / \hbar}}{\sqrt{2 \pi}} \Gamma\left(\frac{1}{2}-i \frac{\mu^{\prime}}{\hbar}\right)\left(\begin{array}{ll}
\mathrm{e}^{\pi \mu^{\prime} / 2 \hbar+i \pi / 4} & \mathrm{e}^{-\pi \mu^{\prime} / 2 \hbar-i \pi / 4} \\
\mathrm{e}^{-\pi \mu^{\prime} / 2 \hbar-i \pi / 4} & \mathrm{e}^{\pi \mu^{\prime} / 2 \hbar+i \pi / 4}
\end{array}\right)
$$

Proof. Let $u \in \mathscr{D}^{\prime}(\mathbb{R})$ be a solution of equation $Q u=\mu^{\prime} u, u$ has to be $\mathscr{C}^{1}$ on $\mathbb{R}_{+}^{*}$ and $\mathbb{R}_{-}^{*}$ (see for instance [Hö], Corollary 3.1.5). So there exists two complex numbers $\alpha_{+}$and $\alpha_{-}$such that the support of $u-\alpha_{+} u_{+}^{0}-\alpha_{-} u_{-}^{0}$ is $\{0\}$, and one sees easily that no finite combination of derivatives of Dirac mass at 0 is a solution of Eq. (27). namely

Writing $v_{+}^{0}$ as the boundary value of an analytic function in the upper half-plane,

$$
v_{+}^{0}\left(x, \mu^{\prime}, \hbar\right)=\lim _{y \rightarrow 0^{+}} \int_{0}^{+\infty} \mathrm{e}^{i(x+i y) \xi / \hbar} \xi^{-1 / 2-i \mu^{\prime} / \hbar} \frac{d \xi}{\sqrt{2 \pi \hbar}},
$$

and with $z=(y-i x) \xi / \hbar$ as a new variable, we get

$$
I_{y}(x)=\frac{\hbar^{-i \mu^{\prime} / \hbar}}{\sqrt{2 \pi}}(y-i x)^{-1 / 2+i \mu^{\prime} / \hbar} \int_{\gamma(y)} \mathrm{e}^{-z} z^{-1 / 2-\imath \mu^{\prime} / \hbar} d z
$$

where $\gamma(y)$ is the image of $[0,+\infty$ [ under $t \mapsto(y-i x) t / \hbar$. Using Cauchy's formula we obtain

$$
v_{+}^{0}\left(x, \mu^{\prime}, \hbar\right)=\frac{\hbar^{-\imath \mu^{\prime} / \hbar}}{\sqrt{2 \pi}} \Gamma\left(\frac{1}{2}-i \frac{\mu^{\prime}}{\hbar}\right)\left(E\left(\mu^{\prime}, \hbar\right) u_{+}^{0}+\frac{1}{E\left(\mu^{\prime}, \hbar\right)} u_{-}^{0}\right),
$$

where $E\left(\mu^{\prime}, \hbar\right)=\mathrm{e}^{2 \pi / 4+\mu^{\prime} \pi / 2 \hbar}$.

We can also write in a similar way $v_{-}^{0}$ as a boundary value of an analytic function in the lower half-plan and get 


$$
v_{-}^{0}\left(x, \mu^{\prime}, \hbar\right)=\frac{\hbar^{-i \mu^{\prime} / \hbar}}{\sqrt{2 \pi}} \Gamma\left(\frac{1}{2}-i \frac{\mu^{\prime}}{\hbar}\right)\left(\frac{1}{E\left(\mu^{\prime}, \hbar\right)} u_{+}^{0}+E\left(\mu^{\prime}, \hbar\right) u_{-}^{0}\right),
$$

and this gives $B\left(\mu^{\prime}, \hbar\right)$. That this matrix is unitary is a direct consequence of the complement formula

$$
\Gamma\left(\frac{1}{2}-i \frac{\mu^{\prime}}{\hbar}\right) \Gamma\left(\frac{1}{2}+i \frac{\mu^{\prime}}{\hbar}\right)=\frac{\pi}{\cosh \left(\pi \mu^{\prime} / \hbar\right)}=\frac{2 i \pi}{E^{2}-E^{-2}}
$$

and this ends the proof.

We shall need below some knowledge about the microsupport of these distributions. Looking at the examples given in Appendix A.1 and using the fact that the canonical transformation associated to the F.I.O. $\mathscr{F}_{\hbar}^{-1} \Gamma$ is the rotation by $\pi / 2$ around $(0,0)$, the reader would easily prove this last

Proposition 12. Let $\sigma_{l, r, u, d}$ be the half-lines in $T^{*} \mathbb{R}$ given by $\sigma_{l}=\mathbb{R}^{-} \times\{0\}, \sigma_{r}=$ $\mathbb{R}^{+} \times\{0\}, \sigma_{u}=\{0\} \times \mathbb{R}^{+}$, and $\sigma_{d}=\{0\} \times \mathbb{R}^{-}$.Then

- MS( $\left.u_{0}^{+}\right)$is a subset of a neighborhood of $\sigma_{r} \cup \sigma_{u} \cup \sigma_{d}$

- MS( $\left.u_{0}^{-}\right)$is a subset of a neighborhood of $\sigma_{l} \cup \sigma_{u} \cup \sigma_{d}$

- MS( $\left.v_{0}^{+}\right)$is a subset of a neighborhood of $\sigma_{u} \cup \sigma_{l} \cup \sigma_{r}$

- $M S\left(v_{0}^{-}\right)$is a subset of a neighborhood of $\sigma_{d} \cup \sigma_{l} \cup \sigma_{r}$

\subsection{Microlocal solutions}

Using the solutions for the model and the reduction theorem (Prop. 8), we define here microlocal solutions of Eq. (27) and study their asymptotic expansion as $\hbar \rightarrow 0$. Because the F.I.O. $U$ only acts on microlocal distributions defined near $(0,0)$ in $T^{*} \mathbb{R}$, we have first to microlocalize the four solutions $u_{ \pm}^{0}$ and $v_{ \pm}^{0}$ near $(0,0)$ that is to truncate them outside a neighborhood of $(0,0)$ and then to consider them as an equivalence class for the relation $M S(u-v) \cap V=\emptyset$, where $V=D\left(0, \epsilon_{0}\right)$ is the neighborhood of $(0,0)$ in $T^{*} \mathbb{R}$ where the point $(4)$ of Proposition 8 holds. For the sake of simplicity we will use the same notations for these microlocal distributions. Now the microlocal distributions $u_{ \pm}$and $v_{ \pm}$given by

$$
u_{ \pm}=U u_{ \pm}^{0} \quad \text { and } \quad v_{ \pm}=U v_{ \pm}^{0}
$$

are microlocal solutions of Eq. (27) in $D\left(0, \epsilon_{0}\right)$. Using the symmetries of operator $U$, we see that we have $v_{-}=\Gamma u_{+}$and $v_{+}=\Gamma u_{-}$because for example

$$
v_{-}=U v_{-}^{0}=U \Gamma \mathscr{F}_{\hbar} u_{-}^{0}=U \Gamma \mathscr{F}_{\hbar}^{-1} \mathscr{F}_{\hbar}^{2} u_{-}^{0}=\Gamma U u_{+}^{0}=\Gamma u_{+}
$$

so that we can study only $u_{+}$and $u_{-}$. Their microsupports are given by Proposition 12 and relation (2) of Proposition 8 concerning the canonical transformation associated to $U$. Denoting by $\sigma_{ \pm}^{l, r}$ the segments in $p^{-1}(0) \cap V$ given by $\sigma_{ \pm}^{l}=\left\{(x, \xi) \in p^{-1}(0) \cap\right.$ $V, x \leq 0, \pm \xi \geq 0\}$ and $\sigma_{ \pm}^{r}=\left\{(x, \xi) \in p^{-1}(0) \cap V, x \geq 0, \pm \xi \geq 0\right\}$, one sees that for $\mu$ small enough $M S\left(u_{+}\right)$stays in a neighborhood of $\sigma_{+}^{l} \cup \sigma_{+}^{r} \cup \sigma_{-}^{r}$ and that $M S\left(u_{-}\right)$ stays in a neighborhood of $\sigma_{+}^{l} \cup \sigma_{-}^{l} \cup \sigma_{-}^{r}$.

We are interested here in the (leading term of the) asymptotic expansion of $u_{+}$ and $u_{-}$. We first recall that for $\mu$ small enough (independently of $\hbar$ ) there exists two 
turning points near 0 , which are real and of opposite sign for $\mu<0$, and complex conjugate for $\mu>0$. We denote by $a(\mu)$ and $b(\mu)$ these turning points, $b(\mu)$ being the one which is positive when $\mu<0$ and with positive imaginary part when $\mu>0$. We will suppose that $\mu$ is small enough (still independently of $\hbar$ ) so that there exists $\left.\epsilon_{1} \in\right] 0, \epsilon_{0}$ [ such that there are no turning points for $\epsilon_{1}<|x|<\epsilon_{0}$, and that the components of $p^{-1}(\mu)$ are not connected in the annulus $\epsilon_{1}<|(x, \xi)|<\epsilon_{0}$ (see Fig. 6 below).

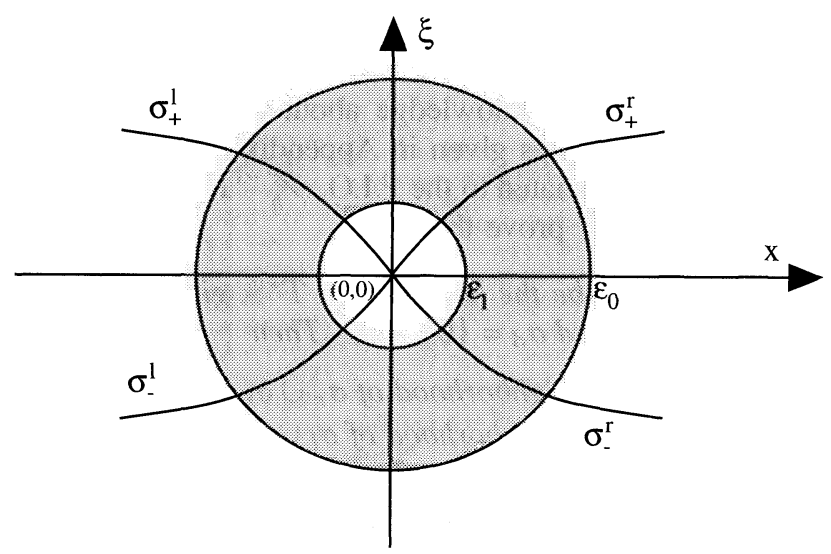

Fig. 6. The critical energy curves

Microlocally in $D\left(0, \epsilon_{0}\right)$, and at the level of principal symbols we have

$$
u_{+}(x, \hbar)=\frac{2^{1 / 4} \mathrm{e}^{i \pi / 8}}{\sqrt{2 \pi \hbar}} \int_{0}^{+\infty} \mathrm{e}^{i \psi(x, y) / \hbar} u_{+}^{0}(y, \hbar) \sigma(x, y, \hbar) d y
$$

Recalling that $\mu-\mu_{0}^{\prime}=O\left(\hbar^{2}\right)$ we have, again at the level of principal symbols,

$$
u_{+}(x, \hbar)=\frac{2^{1 / 4} \mathrm{e}^{\imath \pi / 8}}{\sqrt{2 \pi \hbar}} \int_{0}^{+\infty} \mathrm{e}^{\imath \Psi(x, y, \mu) / \hbar} \frac{d y}{\sqrt{y}},
$$

where $\Psi(x, y, \mu)=\psi(x, y)+\mu_{0}^{\prime} \log y$. We will perform a stationary phase expansion for this integral. We have first the

Proposition 13. For $x \in] \epsilon_{1}, \epsilon_{0}[$, the function $\Psi(x, y, \mu)$ has only one critical point $y_{c}(x, \mu)$ with respect to $y$ satisfying

$$
y_{c}(x, 0)=\sqrt{2} x+O\left(x^{2}\right)
$$

The corresponding critical value $\phi(x, \mu)=\Psi\left(x, y_{c}(x, \mu), \mu\right)$ is given by

$$
\phi_{+}(x, \mu)=\int_{b(\mu)}^{x}\left(2\left(\mu-V_{0}(t)\right)\right)^{1 / 2} d t+\phi_{+}(b(\mu), \mu),
$$

where

$$
\phi_{+}(b(\mu), \mu)=\left\{\begin{array}{ll}
\frac{1}{2} \mu_{0}^{\prime}\left(\log \mu_{0}^{\prime}-1+i \pi\right) & \text { for } \mu>0 \\
\frac{1}{2} \mu_{0}^{\prime}\left(\log \left|\mu_{0}^{\prime}\right|-1\right) & \text { for } \mu<0
\end{array} .\right.
$$


Proof. Let $x$ be inside $] \epsilon_{1}, \epsilon_{0}[$. The critical points of the phase are given by

$$
\partial_{y} \psi(x, y)-\frac{\mu_{0}^{\prime}}{y}=0 .
$$

For $\mu=0$ one has $\mu^{\prime}=0$ and with point (1) of Proposition 9 we see that there is only one critical point $y_{c}(x)$ satisfying $y_{c}(x)=\sqrt{2} x+O\left(x^{2}\right)$. Then we apply the implicit function theorem to the equation $\partial_{y} \Psi(x, y, \mu)=0$ near $\mu=0$ and get a unique function $y_{c}(x, \mu)$ satisfying $y_{c}(x, 0)=y_{c}(x)$ and

$$
-y_{c}(x, \mu) \partial_{y} \psi\left(x, y_{c}(x, \mu)\right)=\mu_{0}^{\prime}
$$

that is

$$
q\left(y_{c}(x, \mu),-\partial_{y} \psi\left(x, y_{c}(x, \mu)\right)\right)=\mu_{0}^{\prime} .
$$

Now we have

$$
\partial_{y} \psi\left(x, y_{c}(x, 0)\right)=O\left(x^{2}\right)
$$

so defining $\eta=-\partial_{y} \psi\left(x, y_{c}(x, \mu)\right)$, we see that $(y, \eta)$ stays in $D\left(0, \epsilon_{0}\right)$, and also that this point is close to the line $\eta=0$ in $T^{*} \mathbb{R}$. Thanks to the relation (3) of Proposition 8 , we have

$$
p\left(x, \partial_{x} \Psi\left(x, y_{c}(x, \mu), \mu\right)\right)=\mu,
$$

so that the critical value $\phi_{+}(x, \mu)=\Psi\left(x, y_{c}(x, \mu), \mu\right)$ satisfies the eikonal equation $p\left(x, \partial_{x} \phi_{+}(x, \mu)\right)=\mu$. Moreover $\xi=\partial_{x} \phi_{+}(x, \mu)$ is positive because $(x, \xi)$ is the image under $\kappa$ of $(y, \eta)$, and $\kappa$ is close to the rotation by $\pi / 4$ around $(0,0)$. So we can write

$$
\phi_{+}(x, \mu)=\int_{b(\mu)}^{x}\left(2\left(\mu-V_{0}(t)\right)\right)^{1 / 2} d t+\phi_{+}(b(\mu), \mu) .
$$

We compute now $\phi_{+}(b(\mu), \mu)$. Because $b(\mu)$ is a turning point we have of course $\partial_{x} \phi(b(\mu), \mu)=0$ and, with $\eta_{c}(\mu)=-\partial_{y} \Psi\left(b(\mu), y_{c}(b(\mu), \mu), \mu\right)$,

$$
y_{c}(b(\mu), \mu) \eta_{c}(\mu)=q\left(y_{c}(b(\mu), \mu), \eta_{c}(\mu)\right)=q \circ \kappa^{-1}(b(\mu), 0)=f_{0} \circ p(b(\mu), 0)=\mu_{0}^{\prime} .
$$

On the other hand we have $\kappa_{\Gamma}(b(\mu), 0)=(b(\mu), 0)$ and, using again relation (5) of Proposition 8,

$$
\kappa\left(y_{c}, \eta_{c}\right)=\kappa_{\Gamma} \circ \kappa\left(y_{c}, \eta_{c}\right)=\kappa \circ \kappa_{A}\left(y_{c}, \eta_{c}\right)=\kappa\left(-\eta_{c},-y_{c}\right)
$$

so we get $y_{c}=-\eta_{c}$ and finally

$$
\left[y_{c}(b(\mu), \mu)\right]^{2}=-\mu_{0}^{\prime}
$$

For $\mu<0$ this gives $y_{c}(b(\mu), \mu)=\sqrt{\left|\mu_{0}^{\prime}\right|}$, and $y_{c}(b(\mu), \mu)=i \sqrt{\mu_{0}^{\prime}}$ for $\mu>0$.

At last we have

$$
\left.\partial_{\mu_{0}^{\prime}} \phi(b(\mu), \mu)=\log y_{c}(b(\mu), \mu)\right)
$$

because $\partial_{y} \Psi\left(x, y_{c}(x, \mu), \mu\right)=0$ by definition of the critical point $y_{c}$, and because $\partial_{x} \Psi\left(b(\mu), y_{c}(b(\mu), \mu), \mu\right)=0$ by definition of the turning point $b(\mu)$. Together with the initial condition $\Psi\left(b(0), y_{c}(0), 0\right)=\psi(0,0)=0$ one gets the value of $\phi(b(\mu), \mu)$ we have announced.

From this proposition, and also with relation (3) of Proposition 9, we immediately get the microlocal value of $u_{+}(x, \hbar)$ near $\sigma_{+}^{r}$ : 
Corollary 2. At the level of principal symbols, and microlocally near $\sigma_{+}^{r}$, we have for $\epsilon_{1}<x<\epsilon_{0}$

$$
u_{+}(x, \hbar)=\frac{2^{1 / 4} \mathrm{e}^{-i \pi / 8}}{\left|y_{c}(x, \mu)\right|^{1 / 2}} \mathrm{e}^{i \phi_{+}(x, \mu) / \hbar} \frac{\sigma_{0}\left(x, y_{c}(x, \mu)\right)}{\left|\partial_{y}^{2} \Psi\left(x, y_{c}(x, \mu), \mu\right)\right|^{1 / 2}} .
$$

We can study the case of

$$
u_{-}(x, \hbar)=\frac{2^{1 / 4} \mathrm{e}^{i \pi / 8}}{\sqrt{2 \pi \hbar}} \int_{-\infty}^{0} \mathrm{e}^{i\left(\psi(x, y)-\mu_{0}^{\prime} \log |y|\right) / \hbar}|y|^{-1 / 2} \sigma_{0}(x, y) d y
$$

along the same lines. We have again one negative critical point $y_{c}(x, \mu)$ satisfying $y_{c}(x, \mu)=\sqrt{2} x+O\left(x^{2}\right)$ and using the same notations we see that $(x, \xi)=$ $\kappa\left(y_{c}(x, \mu), \eta_{c}(x, \mu)\right)$ is near $\sigma_{-}^{l}$, so that we can write for the corresponding critical value

$$
\phi_{-}(x, \mu)=-\int_{a(\mu)}^{x}\left(2\left(\mu-V_{0}(t)\right)\right)^{1 / 2} d t+\phi_{-}(a(\mu), \mu)
$$

when $-\epsilon_{1}>x>-\epsilon_{0}$. We also reproduce the same discussion for the computation of $\phi_{-}(a(\mu), \mu)$ and get that

$$
\phi_{-}(a(\mu), \mu)=\phi_{+}(b(\mu), \mu) .
$$

Then, for any $x \in]-\epsilon_{0},-\epsilon_{1}[$, at the level of principal symbols and microlocally near $\sigma_{-}^{l}$, we have

$$
u_{-}(x, \hbar)=\frac{2^{1 / 4} \mathrm{e}^{-i \pi / 8}}{\left|y_{c}(x, \mu)\right|^{1 / 2}} \mathrm{e}^{i \phi_{-}(x, \mu) / \hbar} \frac{\sigma_{0}\left(x, y_{c}(x, \mu)\right)}{\left|\partial_{y}^{2} \Psi\left(x, y_{c}(x, \mu), \mu\right)\right|^{1 / 2}} .
$$

\section{Microlocal Connection Formulas}

The last step of the proof consists in connecting microlocally near $(0,0)$ the microlocal solutions $u_{ \pm}$and $v_{ \pm}$on one hand, and the complex WKB solutions $\tilde{w}_{ \pm}^{l, r}$ on the other. In fact, as in Sect. 4, we will use the four solutions $w_{ \pm}^{l, r}=w_{ \pm}^{l, r}\left(x, E, h ; x_{0}^{l, r}\right)$ defined by Eq. (22) for suitable choices of $x_{0}^{l, r}$. In order to perform this we only have to remark that the functions $w_{ \pm}^{l, r}$, though they are defined in $\Omega^{l, r}(E)$ can be extended in $\mathscr{S}$ as analytic functions, solutions of the Schrödinger equation. The microsupport of these solutions is given by Propositions 15 and 17 and we see that $M S\left(w_{+}^{l}\right)$ lies in a neighborhood of $\sigma_{+}^{l} \cup \sigma_{+}^{r} \cup \sigma_{-}^{r}$, as $M S\left(w_{+}^{r}\right)$ lies in a neighborhood of $\sigma_{+}^{l} \cup \sigma_{-}^{l} \cup \sigma_{+}^{r}$. The microsupports of $w_{-}^{l}$ and $w_{-}^{r}$ are also given by the relations $w_{-}^{l, r}=\left(w_{+}^{l, r}\right)^{*}=\Gamma w_{+}^{l, r}$.

Microlocally near $\sigma_{-}^{l}$ the solutions $w_{-}^{l}$ and $u_{-}$are "proportional" because these two functions are WKB solutions of the same equation near a simple characteristic (see Proposition 18). More precisely we have

Lemma 4. Let $w_{-}^{l}(x, E)=w_{-}^{l}(x, E, h, a(E))$ be the solution of the Schrödinger equation constructed in Part 3 with $x_{0}=a(E)$ as origin for the phase. Then, microlocally near $\sigma_{-}^{l}$, and for $-\epsilon_{0}<x<-\epsilon_{1}$ we have,

$$
w_{-}^{l}(x, E)=\alpha_{-}^{l}(\mu, h) u_{-}(x, \mu, \hbar)
$$


with

$$
\alpha_{-}^{l}(\mu, h)=\lambda_{+}(\mu, h) \mathrm{e}^{-\imath \phi_{-}(a(\mu), \mu) / \hbar},
$$

where $\lambda_{+}(\mu, h)$ is a classical analytic symbol of order 0 whose principal symbol is $2^{1 / 4} \mathrm{e}^{i \pi / 8} / \sqrt{k}$.

Proof. We only recall that

$$
w_{-}^{l}(x, E)=(V(x)-E)^{-1 / 4} \mathrm{e}^{-z(x, E, a(\mu)) / h}(1+O(h))
$$

and that, microlocally near $\sigma_{+}^{l}$,

$$
u_{-}(x, \mu)=\frac{2^{1 / 4} \mathrm{e}^{-i \pi / 8}}{\left|y_{c}(x, \mu)\right|^{1 / 2}} \mathrm{e}^{i \phi_{-}(x, \mu) / \hbar}\left(\frac{\sigma_{0}\left(x, y_{c}(x, \mu)\right)}{\left|\partial_{y}^{2} \Psi\left(x, y_{c}(x, \mu), \mu\right)\right|^{1 / 2}}+O(\hbar)\right)
$$

so the lemma is obvious concerning the phase of $\alpha_{-}^{l}(\mu, h)$, and its principal symbol is given letting $\mu \rightarrow 0$.

The same arguments lead to the

Lemma 5. Let $w_{+}^{r}(x, E)=w_{+}^{r}(x, E, h, b(E))$ be the solution of the Schrödinger equation constructed in Part 3 with $x_{0}=b(E)$ as origin for the phase. Then, microlocally near $\sigma_{+}^{r}$, and for $\epsilon_{1}<x<\epsilon_{0}$ we have,

$$
w_{+}^{r}(x, E)=\alpha_{+}^{r}(\mu, h) u_{+}(x, \mu, \hbar)
$$

with

$$
\alpha_{+}^{r}(\mu, h)=\rho_{+}(\mu, h) \mathrm{e}^{-i \phi_{+}(b(\mu), \mu) / \hbar},
$$

where $\rho_{+}(\mu, h)$ is a classical analytic symbol of order 0 whose principal symbol is $2^{1 / 4} \mathrm{e}^{2 \pi / 8} / \sqrt{k}$.

We have now in hands all the bricks that make the computation of the scattering matrix (up to an exponentially small error) an elementary algebraic calculation. In fact we can write our three WKB solutions $w_{+}^{l}, w_{-}^{l}$ and $w_{+}^{r}$ (defined at the end of Sect. 3) in the basis $\left(u_{+}, u_{-}\right)$, and then use Proposition 6 to translate the results in terms of the Jost solutions $\tilde{w}_{+}^{l}, \tilde{w}_{-}^{l}$ and $\tilde{w}_{+}^{r}$.

To make the ideas clearer we will write the branching matrix as

$$
B(\mu, \hbar)=\left(\begin{array}{ll}
B_{11} & B_{12} \\
B_{21} & B_{22}
\end{array}\right)
$$

First we have microlocally near $(0,0)$

$$
w_{-}^{l}=\alpha_{+}^{l} u_{+}+\alpha_{-}^{l} u_{-}=\beta_{+}^{l} v_{+}+\beta_{-}^{l} v_{-} .
$$

In this equality we know $\alpha_{-}^{l}$ by Lemma 4 and, because $w_{-}^{l}$ and $v_{-}$have no microsupport near $\sigma_{+}^{l}$, we also have $\beta_{+}^{l}=0$. Proposition 11 gives then

$$
\left(\begin{array}{c}
\alpha_{+}^{l} \\
\alpha_{-}^{l}
\end{array}\right)=B_{\mu^{\prime}}(\hbar)\left(\begin{array}{c}
\beta_{+} \\
\beta_{-}
\end{array}\right)
$$

so that 


$$
\beta_{-}^{l}=\frac{1}{B_{22}} \alpha_{-}^{l} \quad \text { and } \quad \alpha_{+}^{l}=\frac{B_{12}}{B_{22}} \alpha_{-}^{l},
$$

and finally

$$
w_{-}^{l}=\frac{B_{12}}{B_{22}} \alpha_{-}^{l} u_{+}+\alpha_{-}^{l} u_{-} .
$$

We also have $w_{+}^{l}=\Gamma w_{-}^{l}$ and Eq. (35) gives, microlocally near $(0,0)$

$$
\begin{aligned}
w_{+}^{l} & =\overline{\beta_{+}^{l}} \Gamma v_{+}+\overline{\beta_{-}^{l}} \Gamma v_{-} \\
& =\overline{\beta_{-}^{l}} u_{+}+\overline{\beta_{+}^{l}} u_{-} \\
& =\overline{\beta_{-}^{l}} u_{+}+0 u_{-}
\end{aligned}
$$

and, at last

$$
w_{+}^{l}=\frac{1}{\overline{B_{12}}} \alpha_{-}^{l} u_{+}+0 u_{-} .
$$

On the other hand, we have microlocally near $(0,0)$

$$
w_{+}^{r}=\alpha_{+}^{r} u_{+}+\alpha_{-}^{r} u_{-}=\beta_{+}^{r} v_{+}+\beta_{-}^{r} v_{-},
$$

where $\alpha_{+}^{r}$ is given in Lemma 5 and $\beta_{-}^{r}=0$ because $w_{+}^{r}$ and $v_{+}$have no microsupport near $\sigma_{-}^{r}$. We compute the two other coefficients by use of the branching matrix and get

$$
\beta_{+}^{r}=\frac{1}{B_{11}} \alpha_{+}^{r} \quad \text { and } \quad \alpha_{-}^{r}=\frac{B_{21}}{B_{11}} \alpha_{+}^{r}
$$

so that

$$
w_{+}^{r}=\alpha_{+}^{r} u_{+}+\frac{B_{21}}{B_{11}} \alpha_{+}^{r} u_{-}
$$

Noticing that $B_{11}=B_{22}, B_{12}=B_{21}$ and $\operatorname{det} B=\frac{B_{11}}{\bar{B}_{11}}$, Eq. (37) (38) and (39) together give after an easy computation and modulo an exponentially small error,

$$
w_{+}^{l}=B_{11} \frac{\overline{\alpha_{-}^{l}}}{\alpha_{+}^{r}} w_{+}^{r}-B_{21} \frac{\overline{\alpha_{-}^{l}}}{\alpha_{-}^{l}} w_{-}^{l} .
$$

Now with Proposition 6 we obtain

$$
\left\{\begin{array}{l}
s_{11}=\mathrm{e}^{\left(z^{r}(b(E), E)-z^{l}(a(E), E)\right) / h} B_{11} \frac{\overline{\alpha_{-}^{l}}}{\alpha_{+}^{r}} \\
s_{21}=-\mathrm{e}^{-2 z^{l}(a(E), E) / h} B_{21} \frac{\overline{\alpha_{-}^{l}}}{\alpha_{-}^{l}}
\end{array}\right.
$$

and the two Lemmas 4 and 5 give modulo exponentially small errors

$$
\left\{\begin{aligned}
s_{11}= & \frac{1}{\sqrt{2 \pi}}(1+h m(h)) \Gamma\left(\frac{1}{2}-i \frac{\mu^{\prime}}{\hbar}\right) \\
& \mathrm{e}^{\pi \mu^{\prime} / 2 \hbar} \mathrm{e}^{\frac{2}{\hbar}\left(2 \operatorname{Re} \phi_{+}(b(\mu), \mu)-\mu^{\prime} \log h\right)} \mathrm{e}^{\left(z^{l}(a(E), E)-z^{r}(b(E), E)\right) / h} \\
s_{21}= & \frac{i}{\sqrt{2 \pi}}\left(1+h m^{\prime}(h)\right) \Gamma\left(\frac{1}{2}-i \frac{\mu^{\prime}}{\hbar}\right) \\
& \mathrm{e}^{-\pi \mu^{\prime} / 2 \hbar} \mathrm{e}^{\frac{2}{\hbar}\left(2 \operatorname{Re} \phi_{+}(b(\mu), \mu)-\mu^{\prime} \log h\right)} \mathrm{e}^{2 z^{l}(a(E), E) / h},
\end{aligned}\right.
$$


where $m(h)$ and $m^{\prime}(h)$ are CAS of non-negative order. For shorter notations, we shall write

$$
z^{l}(a(E), E)-z^{r}(b(E), E)=i T(E) \quad \text { and } \quad z^{l}(a(E), E)=i T_{-}(E)
$$

and we have as in Sect. 4,

$$
T(E)= \begin{cases}\sqrt{E}(a(E)-b(E))+\int_{-\infty}^{a(E)} Q(t, E) d t+\int_{b(E)}^{+\infty} Q(t, E) d t & \text { for } E<V_{0} \\ \int_{-\infty}^{+\infty} Q(t, E) d t+i \int_{a(E)}^{b(E)}(V(t)-E)^{1 / 2} d t & \text { for } E>V_{0}\end{cases}
$$

and that

$$
T_{-}(E)= \begin{cases}\sqrt{E} a(E)+\int_{-\infty}^{a(E)} Q(t, E) d t & \text { for } E<V_{0} \\ \int_{-\infty}^{0} Q(t, E) d t-i \int_{0}^{a(E)}(V(t)-E)^{1 / 2} d t & \text { for } E>V_{0}\end{cases}
$$

with $Q(t, E)=\sqrt{E-V(t)}-\sqrt{E}$ for real, large enough $t$. It is also necessary to recall here that $\mu^{\prime}=\mu_{0}^{\prime}+O\left(h^{2}\right)$ and

$$
\frac{\pi \mu_{0}^{\prime}}{\hbar}=-\frac{S(\mu)}{\hbar}=-\frac{S(E)}{h}
$$

where $S(\mu)$ is defined in Proposition 10 and $S(E)$ in Eq. (1). We also recall that $S(E)$ is real for any (small enough) real value of $E-V_{0}$, positive for $E<V_{0}$ and negative when $E>V_{0}$. We obtain then Theorem 2 using Prop. 10, 13, Eq. (34) and Eq. (41).

We are now interested in resonances for our equation, that we may define here as poles of the meromorphic continuation of the scattering matrix for complex $E$. The first remark is that if one allows $E$ to have a small imaginary part, that is precisely $|\operatorname{Im} E|<C h$, the solutions for the model operator are still distributions of temperate growth with respect to $h$ as $h \rightarrow 0$, and all we have done in Sect. 5 and Sect. 6 can still be read with no change in that case. To get the remaining theorem we only have to notice that the scattering matrix may be written as

$$
\mathbb{S}(E, h)=\Gamma\left(\frac{1}{2}+i \frac{S(E)}{\pi h}\left(1+h^{2} m_{1}(h)\right)\right) \mathbb{A}(E, h)+O\left(\mathrm{e}^{-\epsilon / h}\right),
$$

where the matrix $\mathbb{A}(E, h)$ is analytic with respect to $\mathrm{E}$ and the error term is uniform with respect to $E$ in a complex neighborhood of $V_{0}$. This shows that the poles of the meromorphic extension of $\mathbb{S}$ are exactly the poles of the gamma function in the first term of the above sum, and this is what we claimed in Theorem 4. 


\section{A. Elements of Semiclassical Microlocal Analysis}

\section{A.1. Sjöstrand spaces}

Definition 4. Let $\Omega$ be an open set in $\mathbb{C}$ and $\Phi$ a continuous function on $\Omega$. The Sjöstrand space $H_{\Phi}(\Omega)$ is the space of functions $f(z, h)$ analytic with respect to $z$ in $\Omega$ and such that, for any compact subset of $\Omega$, there exists $C, \epsilon>0$ such that

$$
\sup \{|f(z, h)|, z \in K\} \leq C \mathrm{e}^{(\Phi(z)+\epsilon) / h} .
$$

Definition 5. Let $f(z, h)$ and $g(z, h)$ be two functions in $H_{\Phi}(\Omega)$ and $\omega$ a subset of $\Omega$. We say that $f \equiv g$ in $\omega$ if, for any compact subset $K$ of $\omega$, there exists $C, \epsilon>0$ such that

$$
\sup \{|f(z, h)-g(z, h)|, z \in K\} \leq C \mathrm{e}^{(\Phi(z)-\epsilon) / h} .
$$

We denote by $\mathscr{H}_{\Phi}(\Omega)$ the corresponding quotient space.

Definition 6. Let $\left(u_{h}\right)_{\left.] 0, h_{0}\right]}$ be a family of compactly supported distributions in $U \subset \mathbb{R}$ whose semi-norms are of order $O\left(h^{-N}\right)$ as $h \rightarrow 0$. The analytic function of $z$ defined by

$$
\mathscr{T} u(z, h)=\int \mathrm{e}^{-(z-x)^{2} / 2 h} u_{h}(x) d x
$$

is called the FBI-transformed of $\left(u_{h}\right)$ and is an element of $H_{(\operatorname{Im} z)^{2} / 2}$.

\section{A.2. Microsupport}

Definition 7. Let $\left(u_{h}\right)_{\left.10, h_{0}\right]}$ be a family of distributions whose semi-norms are of temperate growth with respect to $h$ as $h \rightarrow 0$. We say that $\left(x_{0}, \xi_{0}\right)$ in $T^{*} \mathbb{R}$ is not in the microsupport of $u_{h}$ if there exists a complex neighborhood $\omega$ of $z_{0}=x_{0}-i \xi_{0}$ such that $\mathscr{T} u(z, h)$ is 0 in $\mathscr{H}_{(\operatorname{Im} z)^{2} / 2}(\omega)$. We denote by $M S\left(u_{h}\right)$ the microsupport of $u_{h}$.

Proposition 14. Let $u$ be a distribution independent of $h$. Then the microsupport of $u$ is

$$
M S(u)=(\operatorname{supp} u \times\{0\}) \cup W F_{a}(u),
$$

where $W F_{a}(u)$ is the analytic wavefront set of $u$.

Example 1. Let $H(x)$ be the Heaviside function given by $H(x)=0$ for $x<0$ and $H(x)=1$ for $x>0$. One has $M S(H)=\{0\} \times \mathbb{R} \cup \mathbb{R}^{+} \times\{0\}$.

Proposition 15. Let $a(x, h)$ be a classical analytic symbol on $\mathbb{R}$, whose principal symbol does not vanish, and $s(x)$ a real-analytic function with $s^{\prime \prime}(x) \neq 0$ whenever $s^{\prime}(x)=0$. Then

$$
M S\left(a(x, h) \mathrm{e}^{i s(x) / h}\right)=\left\{(x, \xi) ; \xi=s^{\prime}(x)\right\} .
$$

Definition 8. We say that two distributions $u_{h}$ and $v_{h}$ are microlocally equal near $\left(x_{0}, \xi_{0}\right)$ if there exists a neighborhood $V$ of $\left(x_{0}, \xi_{0}\right)$ in $T^{*} \mathbb{R}$ such that

$$
M S(u-v) \cap V=\emptyset .
$$


Definition 9. A microlocal distribution defined near $\left(x_{0}, \xi_{0}\right)$ is an equivalence class of compactly supported near $x_{0}$ distribution for the microlocal equality near $\left(x_{0}, \xi_{0}\right)$. We denote by $\mathscr{M}\left(x_{0}, \xi_{0}\right)$ the corresponding quotient space, and the notion of microsupport is still relevant in that space.

Proposition 16. Let $P(x, h D)$ be an h-differential operator with analytic coefficients. If $M S(P u)=\emptyset$, then $M S(u) \subset C$ har $P$, where Char $P$ is the set where the principal symbol of $P$ vanishes.

\section{A.3. Microlocal F.I.O}

We will not develop here all the theory of microlocal F.I.O. and the reader should refer to [Sj2] or [De] for a complete description, and to [He-Sj] or [Mä] for an overview. The only few things the reader has to know is that one can define operators of the form

$$
A u(x, h)=\iint \mathrm{e}^{i G(x, y, \theta) / h} a(x, y, \theta, h) u(y, h) d y d \theta
$$

where $a(x, y, \theta, h)$ is a classical analytic symbol and the integration is performed along a suitable contour, acting continuously from one $S$ jöstrand space $\mathscr{H}_{\Phi}$ to another one. An operator of this kind is often called a quantized canonical transformation or here a microlocal F.I.O. because we regard them as operators acting on microlocal distributions. In fact the FBI transformation $\mathscr{T}$ above is 1 to 1 between $\mathscr{H}$ and the corresponding $\mathscr{H}$, so possesses a left-inverse $\mathscr{S}$, and one identify the quantized canonical transformation $A$ with $\mathscr{S} A \mathscr{T}$.

Definition 10. We say that two microlocal F.I.O. $P$ and $Q$ defined in a neighborhood $V$ of $\left(x_{0}, \xi_{0}\right)$ are microlocally equal if for any microlocal distribution $u$ defined in $V$ one has $P u=Q u$ in $\mathscr{M b}(V)$.

Proposition 17. Let $A$ be a microlocal F.I.O. and $\kappa$ its canonical transformation. If $u$ is a microlocal distribution, the microsupport of $A u$ is the image under $\kappa$ of the microsupport of $u$.

Using the well-known fact that an analytic $h$-differential operator of principal type is microlocally equal to $h D_{x}$ we have finally

Proposition 18. Let $P$ be an analytic h-differential operator and $\gamma$ a simple characteristic for $P$. Let $u_{h}$ and $v_{h}$ be two microlocal distributions defined near $\gamma$ such that $M S(P u)=\emptyset, M S(P v)=\emptyset$ and $M S(u) \cap \gamma \neq \emptyset$. Then there exists a classical analytic symbol $\alpha(h)$ and a real constant $\phi$ such that microlocally near $\gamma, v=\alpha \mathrm{e}^{i \phi / h} u$.

\section{References}

[Ag-Kl] Agmon, S., Klein, M.:Analyticity properties in scattering and spectral theory for Schrödinger operators with long-range radial potentials., Duke Mathematical Journal, 68 (2), 337-399 (1992)

[As-Du] Asch, J., Duclos, P.: An elementary model of dynamical tunneling. Differential equations with applications to mathematical physics, Mathematical Science Engineering 192, Boston, MA: Academic Press, pp.1-11 (1993) 
[Br-Co-Du] Briet, P., Combes, J.-M., Duclos, P.: On the location of resonances for Schrödinger operators in the semiclassical limit II: Barrier top resonances. Comm. in Partial Differential Equations, 12 (2), 201-222 (1987)

[Co-Pa] Colin de Verdière, Y., Parisse, B.: Equilibre instable en régime semi-classique I: Concentration microlocale. Prépublication de l'Institut Fourier n.252, 1993

[De] Delort, J.-M.: F.B.I. transformation. Lecture Notes in Maths 1522, Berlin, Heidelberg, New York: Springer, 1993

[Ec] Ecalle, J.: Les Fonctions résurgentes, Publications Mathématiques d'Orsay 81-05, 1981

[Ev-Fe] Fedoryuk, M.V.: Asymptotic behavior as $\lambda \rightarrow \infty$ of the solution of the equation $w^{\prime \prime}(z)-$ $p(z, \lambda) w(z)=0$ in the complex $z$-plane. Russ. Math. Surv., 21, 1-48 (1966)

[Fe] Fedoryuk, M.V.: One-dimensional scattering in the quasiclassical approximation. Differential Equations 1, 483-495,1201-1210 (1965)

[Fr-Fr] Fröman, N., Fröman, P.O.: JWKB approximation. Amsterdam: North-Holland (1965)

[Ge-Gr] Gérard, C., Grigis, A.: Precise estimates of tunneling and eigenvalues near a potential barrier., J. Differ. Eqs. 42, 149-177 (1988)

[Gr] Grigis, A.: Estimations asymptotiques des intervalles d'instabilité pour l'équation de Hill., Ann. Sc. Ecole Normale Supérieure, 4-ième série, 20, 641-672 (1987)

[Hd] Heading, J.: An introduction to phase-integral methods., Methuen, 1962

[He-Sj] Helffer, B., Sjöstrand, J.: Semiclassical analysis of Harper's equation III. Bull. Soc. Math. France, Mémoire 39, 1990

[Hö] Hörmander, L.: The analysis of linear partial differential operators I. Grundlheren der mathematishen Wissenschaften 256, Berlin, Heidelberg, New York: Springer, 1985.

[Je] Jeffreys, H.:On the use of asymptotic approximations of Green's type when the coefficients has zeros. Proc. Cambridge Philos. Soc. 52, 61-66 (1956)

[La] Langer, R.E.:On the connection formulas and the solutions of the wave equation. Phys. Rev. 51, 669-676 (1937)

[Mä] März, C.: Spectral asymptotics for Hill's equation near the potential maximum. Asymptotic Analysis 5, 221-267 (1992)

[Na] Nakamura, S.:Tunneling effects in momentum space and scattering. Lecture Notes in Pure Appl. Math. 161 (Spectral and Scattering Theory, ed. M. Ikawa), New York: Marcel Decker, 1994

[O11] Olver, F.W.J.:Error analysis of phase-integral methods II. Application to wave penetration problems. J. Res. Nat. Bur. Standards, Sect.B69, 291-300 (1965)

[O12] Olver, F.W.J.:Asymptotic and special functions. New York: Academic Press, 1974

[Ra] Ramond, T.: Intervalles d'instabilité pour une équation de Hill à potentiel méromorphe. Bull. Soc. Math. France, 121, 403-444 (1993)

[Sj1] Sjöstrand, J.: Semiclassical resonances generated by non-degenerate critical points. Lecture Notes in Maths 1256, Berlin, Heidelberg, New York: Springer, 1987 , pp.402-429

[Sj2] Sjöstrand, J.: Singularités analytiques microlocales, Astérisque 95 (1982)

[Sj3] Sjöstrand, J.: Density of states oscillations for magnetic Schrödinger operators. Proceedings of the International Conference at Birmingham (Al), USA, 1990, Mathematical Science Engineering 186, Boston, MA: Academic Press, pp.295-345 (1992)

[Vo] Voros, A.:The Return of the Quartic Oscillator. The Complex WKB Method. Ann. Inst. H. Poincaré Phys. Théor., 39(3) (1983)

\section{Communicated by B. Simon}

This article was processed by the author using the $\mathrm{LAT}_{\mathrm{E}} \mathrm{X}$ style file pljourl from Springer-Verlag. 